\title{
Immune dysregulation in SHARPIN-deficient mice is dependent on CYLD-mediated cell death
}

\author{
Rosalind L. Ang ${ }^{*}$, John P. Sundberg ${ }^{2}$, Shao-Cong Sun ${ }^{3}$, Virginia L. Gillespie ${ }^{4}$, Peter S. Heeger ${ }^{1,5}$, \\ Huabao Xiong ${ }^{1}$, Sergio A. Lira ${ }^{1,6}$ and Adrian T. Ting ${ }^{1,6,7^{*} \#}$
}

${ }^{1}$ Precision Immunology Institute, Icahn School of Medicine at Mount Sinai, New York, NY 10029, USA

2 The Jackson Laboratory, Bar Harbor, ME 04609

${ }^{3}$ Department of Immunology, MD Anderson Cancer Center, The University of Texas, Houston, TX 77030

${ }^{4}$ Center for Comparative Medicine and Surgery, Icahn School of Medicine at Mount Sinai, New York, NY 10029, USA

${ }^{5}$ Department of Medicine, Translational Transplant Research Center, Recanati Miller Transplant Institute, Icahn School of Medicine at Mount Sinai, New York, NY 10029, USA

${ }^{6}$ Tisch Cancer Institute, Department of Medicine, Icahn School of Medicine at Mount Sinai, New York, NY 10029, USA

${ }^{7}$ Current address: Department of Immunology, Mayo Clinic, Rochester, MN 55905, USA

*Corresponding authors: angrosalind@gmail.com (R.L.A.); ting.adrian@mayo.edu (A.T.T.)

\# Primary contact: Mayo Clinic, Department of Immunology, 200 First St SW, Rochester, MN 55905.

Tel: +1-507-422-6538. Email: ting.adrian@mayo.edu 
Running title: CYLD-dependent cell death causes inflammation.

Short Summary: In the absence of SHARPIN, cells fail to properly regulate the deubiquitinase CYLD, leading to RIPK1-mediated cell death. Deletion of Cyld reverses the sensitivity of Sharpin ${ }^{-/}$cells to

TNF-induced cell death, as well as the multi-organ inflammation and immune dysfunction observed in Sharpin $^{-/-}$mice. 


\section{Abstract}

SHARPIN, together with RNF31/HOIP and RBCK1/HOIL1, form the linear ubiquitin chain assembly complex (LUBAC) E3 ligase that catalyzes M1-linked poly-ubiquitination. Mutations in

$4 \quad$ RNF31/HOIP and RBCK/HOIL1 in humans and Sharpin in mice lead to auto-inflammation and

5 immunodeficiency but the mechanism underlying the immune dysregulation remains unclear. We now

6 show that the phenotype of the Sharpin ${ }^{-/}$mice is dependent on CYLD, the deubiquitinase that removes

7 K63-linked poly-ubiquitin chains. The dermatitis, disrupted splenic architecture, and loss of Peyer's

8 patches in the Sharpin $^{-/-}$mice were fully reversed in Sharpin ${ }^{-/} C y l d^{-/}$mice. There is enhanced association

9 of RIPK1 with the death-inducing signaling complex (DISC) following TNF stimulation in Sharpin ${ }^{-/-}$

10 cells, and this is dependent on CYLD since it is reversed in Sharpin ${ }^{-/-} \mathrm{Cyld}^{-/-}$cells. Enhanced RIPK1

11 recruitment to the DISC in Sharpin ${ }^{-/}$cells correlated with impaired phosphorylation of CYLD at serine

12418 , a modification reported to inhibit its enzymatic activity. The dermatitis in the Sharpin ${ }^{-/}$mice was

13 also ameliorated by the conditional deletion of Cyld using LysM-cre or Cx3crl-cre indicating that

14 CYLD-dependent death of myeloid cells is inflammatory. Our studies reveal that under physiological

15 conditions, TNF- and RIPK1-dependent cell death is suppressed by the linear ubiquitin-dependent

16 inhibition of CYLD. The Sharpin ${ }^{-/}$phenotype illustrates the pathological consequences when CYLD

17 inhibition fails. 


\section{Introduction}

Linear or M1-linked poly-ubiquitin modifications play a major role in regulating immune responses (1-3). This modification is catalyzed by the linear ubiquitin chain assembly complex (LUBAC) E3 ligase, which consists of the catalytic component RNF31/HOIP in complex with two other essential proteins with non-catalytic functions RBCK1/HOIL1 and SHARPIN (4-10). Linear ubiquitination plays a key role in signaling downstream of several immune receptors including Tumor Necrosis Factor Receptor Superfamily (TNFRSF) members, antigen receptors and pattern recognition receptors $(1,11)$. In the case of TNF $\alpha$ (TNF), LUBAC components are rapidly recruited to TNFR1

9 following receptor ligation to ubiquitinate target molecules, providing scaffolding for the formation of 10 multi-molecular complexes that propagate signaling. One critical signaling complex assembled by linear

11 ubiquitin is the $\mathrm{I}-\kappa \mathrm{B}$ kinase (IKK) complex that phosphorylates $\mathrm{I}-\kappa \mathrm{B} \alpha(5,6,12)$, a step required for

12 subsequent degradation of $\mathrm{I}-\kappa \mathrm{B} \alpha$ and induction of $\mathrm{NF}-\kappa \mathrm{B}$-dependent gene transcription. NEMO

13 (encoded by the Ikbkg gene), a structural component of the IKK complex, is modified by linear

14 ubiquitination and also possesses a linear ubiquitin-binding domain that facilitates oligomerization of the 15 IKK complex $(5,6,12,13)$. Cells deficient in linear ubiquitination have impaired IKK activity and NF-

$16 \kappa \mathrm{B}$ signaling in response to TNF stimulation $(5,7-9)$. Since IKK can phosphorylate substrates other than

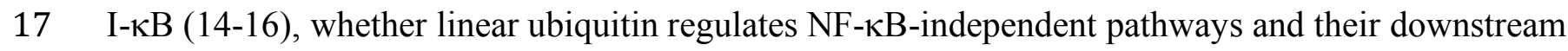

18 biological processes remains poorly understood. Consistent with its role in the signaling of immune receptors, LUBAC components are critical

20 for proper immune regulation. Loss-of-function mutations in $R N F 31 / H O I P$ and in $R B C K 1 / H O I L 1$ in

21 humans cause immunodeficiency, autoinflammation, amylopectinosis, and lymphangiectasia $(17,18)$. In

22 mice, a loss-of-function mutation in Sharpin has been found in the chronic proliferative dermatitis

$23(\mathrm{cpdm})$ mice (hereafter referred to as $\mathrm{Sharpin}^{-/}$), which spontaneously develops dermatitis, multi-organ

24 inflammation and displays immunodeficiency (7-9, 19-21). Crossing of Sharpin ${ }^{-/}$mice with Tnf $^{/-}$mice 
reversed the skin inflammation indicating that some of the lesion in this mutant mouse are TNF-driven

(9). Sharpin'-- cells exhibited enhanced cell death in response to TNF $(8,9)$ suggesting that the

phenotype of the Sharpin ${ }^{-/}$mice could be due to inappropriate cell death. Subsequent genetic crosses

with knockouts of death-signaling molecules provide further support for this hypothesis. Deletion of the

necroptosis-signaling molecule Ripk3 delayed but did not prevent the dermatitis but additional deletion of a single allele of the apoptosis-signaling molecule Casp8 or skin-specific deletion of Fadd reversed the dermatitis in the Sharpin'-- mice $(22,23)$. Furthermore, crossing with a kinase-inactive Ripk1 ${ }^{\text {tm } 1.1 \text { Gsk }}$ $\left(\right.$ Ripk1 $\left.{ }^{K 45 A}\right)$ allele also reversed the phenotype of the Sharpin ${ }^{-/}$mice (24). These genetic analyses suggest that the phenotype of the Sharpin ${ }^{-/}$mouse is due to excessive RIPK1-dependent cell death and we recently suggested the term ripoptocide to denote this manner of cell death orchestrated by RIPK1 (25).

11 One mechanism by which Sharpin deficiency could lead to aberrant RIPK1 death-signaling is impaired

12 phosphorylation of RIPK1 catalyzed by members of the IKK family, which normally inhibits its death-

13 promoting activity (26-30). Whether the Sharpin deficiency also affects other molecules involved in

14 regulating cell death is unclear.

Another form of poly-ubiquitin modification, one in which the covalent linkage occurs at K63 of 16 the ubiquitin molecule, is also critical in TNF signaling $(31,32)$. This form of ubiquitin modification is

17 mediated by TRAF2-dependent recruitment of the cIAP1/2 E3 ligases (encoded by Birc2 and Birc3) and 18 one key molecule modified in this manner is RIPK1. Cells in which the ubiquitin acceptor site of RIPK1 19 at K377 was mutated were more sensitive to TNF-mediated apoptosis, similar to cells in which TRAF2 20 and cIAP1/2 were inhibited (33-35). Cells treated with Second Mitochondria-Derived Activator Of

21 Caspase (SMAC) mimetics, which induce the degradation and loss of cIAP1/2, displayed reduced poly-

22 ubiquitination of RIPK1 $(34,35)$. This cell protective function of K63-linked poly-ubiquitination of

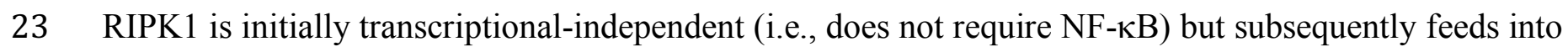

24 NF-кB-dependent induction of pro-survival genes. A unifying model proposes that there are two cell 
1 death checkpoints in the TNFR1 signaling pathway. The early checkpoint (Checkpoint 1) prevents

2 RIPK1 from becoming a death-signaling molecule via K63-linked poly-ubiquitination, which is

3 followed by the NF-kB-dependent induction of pro-survival genes during the late checkpoint

4 (Checkpoint 2) (36-38). Disruption of Checkpoint 1 is often achieved in vitro by blocking RIPK1

5 ubiquitination using SMAC mimetics, which leads to CASP8-dependent apoptosis or RIPK3/MLKL-

6 dependent necroptosis (35, 39-41). The relationship between linear and K63 ubiquitin linkages in

7 regulating cell death and potentially in the immune dysfunction observed in LUBAC deficiencies is

8 currently unclear.

We now report that the molecule CYLD is central to the immunopathology observed in the

10 Sharpin $^{--}$mice. CYLD is a deubiquitinase that dismantles K63-linked poly-ubiquitin chains (42) and its

11 activity was shown to be suppressed by IKK $\beta$-mediated phosphorylation (43). CYLD has also been

12 reported to associate with RNF31/HOIP via SPATA2 (44-48). We found that SHARPIN-deficient cells,

13 which are deficient in IKK activity, display reduced CYLD phosphorylation following receptor ligation.

14 Coincident with defective CYLD phosphorylation in the SHARPIN-deficient cells, there is enhanced

15 association of its substrate RIPK1 with a death-inducing signaling complex (DISC) to induce apoptosis

16 and necroptosis, which is reversed in SHARPIN and CYLD double-deficient cells. The phenotype of the

17 Sharpin ${ }^{-/}$mice is reversed by a compound deletion of Cyld and importantly, conditional deletion of Cyld

18 in myeloid cells significantly reversed the dermatitis. Our study provides evidence that linear ubiquitin-

19 dependent suppression of CYLD is one mechanism that keeps ripoptocide at bay and to maintain

20 immune homeostasis, disruption of which leads to immune dysfunction. 


\section{Results}

\section{Cyld is essential for the development of inflammation in Sharpin-deficient mice.}

Sharpin $^{-/}$cells exhibited heightened sensitivity to TNF-induced killing (9). Since CYLD has been reported to be required for TNF to induce RIPK1-dependent apoptosis and necroptosis $(34,49-53)$, we asked if CYLD has any role in the sensitivity of Sharpin-- cells to TNF-induced cell death. We first confirmed that Sharpin $^{-/}$mouse embryonic fibroblasts (MEF) were more sensitive to TNF-induced necroptosis than their wild type counterparts, an effect that was reversed when the Sharpin ${ }^{-/-}$MEF were complemented with Sharpin but not with a control gene (Supplementary Figure S1A). Comparison of the Sharpin ${ }^{-/}$MEF complemented with Sharpin versus the control gene showed SHARPIN-deficient

10 cells to be more sensitive to TNF-induced necroptosis in a dose and time-dependent manner

11 (Supplementary Figure S1B-D). Furthermore, when both SHARPIN-sufficient and SHARPIN-deficient

12 MEF were stably transfected with the non-degradable I $\kappa$ B super-repressor (I $\kappa$ BSR) to block NF- $\kappa$ B

13 induction, SHARPIN-deficient MEF remained more sensitive to death compared to SHARPIN-

14 sufficient MEF (Supplementary Figure S1E). Blotting of nuclear extracts from the Sharpin-

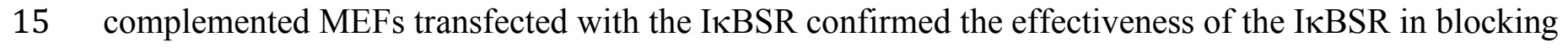

16 translocation of the NFkB p65 subunit to the nucleus (Supplementary Figure S1F). This indicated that

17 the loss of SHARPIN could sensitize cells to death through a NF- $\kappa$ B-independent mechanism,

18 reminiscent of the effect of mutating the acceptor site on RIPK1 for K63-linked poly-ubiquitin (33). As

19 an initial test of a potential role for CYLD, we examined the effect of knocking down CYLD on

20 necroptosis in SHARPIN-deficient MEF. Knocking down CYLD significantly reduced the level of

21 necroptosis in response to TNF (Supplementary Figure S1G \& H). This observation suggested that the

22 loss of SHARPIN led to CYLD-dependent death.

These results prompted us to test whether CYLD regulates the phenotype of the Sharpin ${ }^{-/}$mice.

24 To test this hypothesis, we generated Sharpin ${ }^{--} C y l d^{--}$double deficient animals. Strikingly, the loss of 
1 Cyld fully prevented the spontaneous development of dermatitis that develops in Sharpin ${ }^{-/}$mice (Figure 1A \&B). Histological analysis showed that ulceration, thickening of the epidermis and leukocytic infiltration observed in Sharpin ${ }^{-/}$skin were absent in Sharpin ${ }^{-/ C}$ Cyld ${ }^{-/}$skin (DKO, Figure 1C). Whereas Sharpin $^{-/}$mice were smaller and weighed less than age-matched wild type mice, sizes and weights of the Sharpin ${ }^{-/} \mathrm{Cyld}^{-/}$mice and the wild type controls did not differ (Figure 1A, Supplementary Figure S2A). The skin of Sharpin $^{--}$mice were reported to be infiltrated by immune cells including granulocytes and macrophages (19). Immunohistochemical analysis confirmed the presence of CD45 ${ }^{+}$hematopoietic cells in the skin from Sharpin'-- mice but not from Sharpin ${ }^{-/} C y l d^{-/}$mice (Figure 1D \& E). F4/80+ macrophages were also detected in the inflamed skin of the Sharpin ${ }^{-/}$mice (Figure 1F \& G). Thus, the dermatitis in the Sharpin ${ }^{-/}$mice was ameliorated by the compound loss of Cyld. Lymphoid organs in Sharpin ${ }^{-/}$mice are known to be abnormal $(20,21)$. Gross examination of

12 immune organs showed that the enlarged spleen, shrunken thymus and mesenteric lymph nodes and the 13 enlarged liver typical of Sharpin ${ }^{-/-}$mice were absent in the Sharpin $^{-/} \mathrm{Cyld}^{-/-}$mice (Figure 2A,

14 Supplementary Figure S2B \& C). We observed no alterations in the weight of the hearts or the length of 15 the colon in any of the genotypes (Supplementary Figure S2D \& E). The splenic architecture of the 16 Sharpin $^{-/}$spleen lacks lymphoid follicles whereas the Sharpin ${ }^{-/} C y l d^{-/}$spleen is similar to that of 17 Sharpin $^{+/+}$spleen and displays no overt defect (Figure 2B). Adult Sharpin ${ }^{-/}$mice have no Peyer's 18 patches in their small intestine $(20,54)$ but these were present in the Sharpin ${ }^{-/}$Cyld ${ }^{-/-}$mice (Figure 2C \& 19 D). Steady state serum immunoglobulin levels are also perturbed in the Sharpin ${ }^{-/}$mice (20). They have

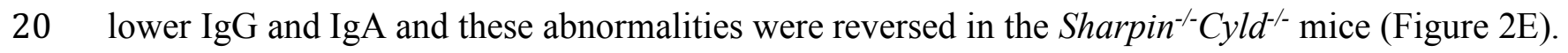

21 Serum IgE level was elevated in the Sharpin ${ }^{-/}$mice but was reversed in the Sharpin ${ }^{-/} C y l d^{-/}$mice

22 (Figure 2E). Consistent with the lack of Peyer's patches, adult Sharpin ${ }^{-/}$mice have no fecal IgA (20),

23 but this was also reversed in the Sharpine- Cyld $^{-/}$mice (Figure 2F). Whereas several cytokines, including 24 IFNG, IL5 and IL12/23p40 were elevated in the serum of Sharpin ${ }^{-/}$mice compared to wild type 
1 controls, these elevations were abrogated in the Sharpin ${ }^{-/} C y l d^{-/}$mice (Figure 2F). More modest changes

2 in serum IL2 and IL6, but not eotaxin and CCL3 (MIP-1 $\alpha$ ), were observed in the Sharpin ${ }^{-/-}$mice

3 (Supplementary Figure S2F-I). Together, these data demonstrate that CYLD is critical for eliciting the

4 multi-organ inflammation and abnormal lymphoid tissues observed in the Sharpin ${ }^{-/}$mice, suggesting

5 dysregulated CYLD activity in the absence of SHARPIN.

\section{CYLD phosphorylation is disrupted by SHARPIN deficiency.}

The above results showed a clear genetic interaction between SHARPIN and CYLD but the mechanistic relationship between the two molecules was unknown. Following TNFR1 ligation, an array

10 of signaling scaffold and effector molecules such as LUBAC, cIAP1/2, TRAF2, and IKK are recruited

11 to TNFR1. This receptor complex, also known as Complex I (55), propagates a survival signal. CYLD

12 can also be recruited to the receptor complex via a SPATA2-dependent association with RNF31/HOIP

13 (44-48). But how defects in linear ubiquitin could lead to CYLD activation was not known. CYLD is

14 regulated by post-translational mechanisms, and cleavage by CASP8 and MALT1 have been reported to

15 regulate its function $(53,56)$. Another potential regulatory mechanism may be phosphorylation. CYLD

16 phosphorylation at a cluster of serine residues around Ser 418 carried out by IKK $\beta$ in a stimulus

17 dependent fashion is known to suppress its enzymatic activity (43). Phosphorylation of CYLD at Ser

18418 by IKK $\varepsilon$ was also reported to have the same effect (57). Since SHARPIN-deficient cells were

19 reported to have diminished IKK activity (8), we postulated that SHARPIN deficiency would also lead

20 to diminished CYLD phosphorylation, which would result in a more active CYLD to initiate cell death.

21 To test this, we examined the phosphorylation kinetics of CYLD in primary adult dermal fibroblast

22 (ADF). Using an antibody that detects phosphorylated Ser 418 of CYLD, we observed an induction in

23 CYLD phosphorylation in Sharpin ${ }^{+/+}$cells but this phosphorylation was diminished in Sharpin ${ }^{-/}$cells

24 (Figure 3A). To confirm that the defect in CYLD regulation is caused specifically by SHARPIN 
deficiency, we examined the SHARPIN-complemented MEF described in Supplementary Figure S1.

2 Complementation of Sharpin ${ }^{--}$cells with Sharpin, but not with a control gene, restored CYLD

3 phosphorylation (Figure 3B). Consistent with the previous study that IKK $\beta$ phosphorylates CYLD (43),

4 blockade of IKK $\beta$ with the highly selective chemical inhibitor [5-( $p$-Fluorophenyl)-2-ureido]thiophene-

5 3-carboxamide (TPCA-1) resulted in reduced CYLD phosphorylation in Sharpin ${ }^{+/}$MEF, similar to that

6 observed in Sharpin $^{-/}$MEF (Figure 3C). To extend our observations beyond fibroblasts, we examined

$7 \quad$ splenic B cells. Sharpin ${ }^{-/-}$B cells also showed diminished CYLD phosphorylation when compared to their wild type counterparts (Figure 3D) though there appears to be some reduction in the level of CYLD

9 in the Sharpin ${ }^{-/}$B cells. Similarly, in total splenocytes, CYLD phosphorylation was detected in

10 Sharpin $^{+/+}$but not in Sharpin ${ }^{-/}$cells (Figure 3E). Interestingly, blotting for CYLD revealed a significant

11 loss in full-length CYLD in the Sharpin ${ }^{-/}$splenocytes, accompanied by the detection of smaller

12 fragments of CYLD. CASP8 and MALT1 have been shown to cleave CYLD $(53,56,58)$. A $25 \mathrm{kD}$ and

$1335 \mathrm{kD}$ N-terminal fragment of CYLD that are products of CASP8- and MALT1-mediated proteolysis,

14 respectively, are detected in the Sharpin $^{-/}$splenocytes. These observations suggest that compensatory

15 mechanisms to remove CYLD may be invoked in the face of SHARPIN deficiency to attain a cellular

16 state akin to deleting Cyld in the Sharpin ${ }^{--} C y l d^{--}$mice. These findings indicate that in Sharpin

17 deficiency, CYLD phosphorylation is impaired resulting in its initiation of cell death and that some cell

18 types may attempt to avoid this fate by removing CYLD through other mechanisms.

\section{SHARPIN-deficient cells are more susceptible to CYLD-mediated cell death.}

Since our earlier result in MEF showed a role for SHARPIN and CYLD in regulating cell death

22 (Supplementary Figure S1), we examined the sensitivity of ADF obtained from Sharpin ${ }^{+/+}$, Sharpin ${ }^{-/}$,

$23 \mathrm{Cyld}^{-/}$and Sharpin ${ }^{-/} \mathrm{Cyld}^{-/-}$mice to TNF-induced cell death. Sharpin ${ }^{-/}$ADF were more susceptible to

24 TNF-induced apoptosis and necroptosis when compared to their Sharpin $^{+/+}$counterpart (Figure 4A). The 
increased susceptibility to both forms of death was abolished in Sharpin ${ }^{-/} C y l d^{-/}$cells (Figure 4A), consistent with observations obtained earlier by knocking down CYLD (Supplementary Figure S1). Similarly, Sharpin ${ }^{-/}$ADF exhibited enhanced apoptotic markers (i.e., cleaved CASP8, CASP3 and PARP) and a necroptotic marker (i.e., phospho-MLKL) when compared to $\operatorname{Sharpin}^{+/+}$ADF, and these death signatures were diminished in the Sharpin $^{--} \mathrm{Cyld}^{--}$ADF (Figure 4B-E). Similar data were also observed in MEF. Sharpin ${ }^{-/}$MEF were more sensitive to apoptosis and necroptosis, which was reversed in the Sharpin ${ }^{--} \mathrm{Cyld}{ }^{-/}$MEF (Supplementary Figure S3A-E). These results demonstrate that enhanced sensitivity to cell death in SHARPIN-deficient cells is dependent on CYLD. CYLD initiates deathsignaling by removing K63-linked ubiquitin chains from RIPK1 thereby converting RIPK1 from a

10 survival-signaling to a death-signaling effector $(31,53,59)$. This conversion is biochemically detected

11 by the translocation of RIPK1 to the FADD/CASP8 death-inducing signaling complex (DISC). We

12 therefore sought to confirm that there was more conversion of RIPK1 to its death-signaling form in

13 SHARPIN-deficient cells and that this was dependent on CYLD. In ADF treated with TNF, in the

14 presence of a combination of cycloheximide and zVAD-fmk to stabilize the DISC, RIPK1 was detected

15 in the FADD pulldowns of Sharpin-/ ADF more rapidly than in Sharpin ${ }^{+/+}$cells (Figure 4F). RIPK1 translocation to the DISC was abrogated in Sharpin $^{-/}$Cyld ${ }^{-/-}$ADF (Figure 4F) demonstrating that CYLD

17 is necessary for the conversion of RIPK1 to a death-signaling molecule when SHARPIN is absent. The

18 dependence on CYLD for RIPK1 translocation to the DISC was also observed in MEF (Supplementary

19 Figure S3F). We confirmed expression, or lack therefore, of SHARPIN and CYLD in the MEF of the

20 indicated genotype (Supplementary Figure S3G). As an additional confirmation, we reconstituted

$21 \mathrm{Sharpin}^{-/} \mathrm{Cyld}{ }^{-/-} \mathrm{MEF}$ with retroviral vectors encoding SHARPIN only, CYLD only or both molecules

22 together (Supplementary Figure S3H \& 3I). These reconstituted MEF showed similar data

23 (Supplementary Figure S3H) to that observed previously in ADF (Figure 4F) and MEF (Supplementary

24 Figure S3F). 
If $\mathrm{Sharpin}^{-/}$cells are more sensitive to death due to defective CYLD phosphorylation, then a non-phosphorylatable CYLD mutant would be predicted to mimic the SHARPIN deficiency (i.e., more sensitive to cell death). Mutations in the cluster of seven serines around residue 418 of human CYLD was shown to result in a gain-of-function in deubiquitinase activity (43). We generated an analogous mutant of mouse CYLD with seven serine to alanine substitutions (CYLD-S7A) and complemented Cyld ${ }^{-/}$MEF with either wild type or mutant CYLD. The complemented cells were treated with TNF in the presence of zVAD-fmk in order to prevent CYLD cleavage by CASP8 (53) and thus negate the potential confounding issue of differential cleavage of CYLD-WT versus CYLD-S7A by CASP8. Under this stimulation condition, CYLD-complemented cells undergo necroptosis (53) and this death response, quantified by PI staining, was enhanced in the CYLD-S7A-complemented cells as compared to CYLD-

11 WT-complemented cells (Figure 4G). The CYLD-S7A-complemented cells phenocopied the SHARPIN-

12 deficient cells (Supplementary Figure S1A \& 1B), consistent with the notion that phosphorylation and

13 therefore inhibition of CYLD, is a key regulatory event that suppresses cell death in response to TNF.

14 Our findings imply that there is dysregulated CYLD activity in SHARPIN-deficient cells.

\section{CYLD-mediated death of myeloid cells causes skin inflammation}

The leukocytic infiltration in the skin of the Sharpin ${ }^{-/}$mice consists of granulocytes and macrophages (19) prompting us to examine the role of macrophages in the pathogenesis of skin lesions

19 in these mice. We recently reported that macrophages are highly susceptible to CYLD-dependent autonecroptosis mediated by the TNF that is produced in response to TLR4 ligation (59). Bone marrow

21 derived macrophages (BMDM) deficient in SHARPIN showed a defect in CYLD phosphorylation when

22 stimulated with TNF (Figure 5A \& Supplementary Figure S4A) albeit the defect was modest. A similar

23 defect was also observed when these cells were stimulated with lipopolysacharrides (LPS) to activate

24 TLR4, or polyIC (pIC) to activate TLR3 (Figure 5B \& C, Supplementary Figure S4B \& C). Therefore, 
we tested to see if these macrophages also exhibit altered necroptosis. In response to low-dose LPS that elicits TNF-dependent necroptosis (59), Sharpin ${ }^{-/}$BMDM were more sensitive to necroptosis compared to their wild type counterparts and this was abrogated in the Sharpin ${ }^{-/-C y l d}{ }^{-/-}$BMDM (Figure 5D). A similar effect was also observed using pIC to trigger necroptosis (Figure 5E). Analysis of DISC

formation showed that RIPK1 was detected in FADD pulldowns of Sharpin ${ }^{-/}$BMDM more rapidly than in Sharpin $^{+/+}$cells treated with TNF (Figure 5F). Similar to that observed previously in ADF (Figure 4F), RIPK1 association with the DISC was abrogated in Sharpin ${ }^{--}$Cyld ${ }^{-/}$BMDM. To confirm the in vitro BMDM data, we looked for evidence of macrophage death in skin sections taken from mice of the different genotypes. Consistent with a previous report showing apoptosis occurring in the skin of Sharpin $^{--}$mice (60), we detected cleaved CASP3 in the skin of Sharpin'-- mice, a proportion of which

11 co-localized with F4/80+ macrophages (Figure 5G \& H). However, cleaved CASP3 was largely absent

12 in the Sharpin ${ }^{-/}$Cyld ${ }^{-/}$skin consistent with our in vitro data showing that SHARPIN-deficient cells

13 undergo cell death in a CYLD-dependent manner.

The genetic crosses conducted using germline knockouts showed CYLD to be the cause of the phenotype observed in Sharpin ${ }^{-/-}$mice. However, the contribution of CYLD-dependent death in specific cellular compartments to the Sharpin ${ }^{-/}$phenotype is not clear. The data in Figure 5A-H prompted us to

17 ask whether CYLD-dependent macrophage death has any role in the pathogenesis of the Sharpin ${ }^{-/}$mice.

18 We crossed the Sharpin-/ strain to the LysM-cre-driven conditional knockout of Cyld $\left(C y l{ }^{M-K O}\right)$ we 19 previously generated (59). The Sharpin ${ }^{-/} C y l d^{M-K O}$ mice showed significant improvement in their

20 dermatitis compared to the Sharpin ${ }^{-/}$mice, which develop discernable dermatitis by week 8 in our

21 colony (Figure 6A). In our cohort, two thirds of Sharpin ${ }^{--} C y l d^{M-K O}$ mice did not show skin inflammation

22 by week 16 while the remaining 1/3 had delayed progression (Supplementary Figure S4D). Histological

23 analysis showed that the typical ulceration, thickening of the epidermis and leukocytic infiltration

24 observed in Sharpin ${ }^{-/}$skin was absent in Sharpin $^{-/} C y l d^{M-K O}$ mice with no disease (Figure 6B). However, 
1 other defects in the spleen and thymus of Sharpin ${ }^{-/-}$mice were not reversed in the Sharpin ${ }^{-/-} C y l d^{M-K O}$

2 mice (Figure 6C \& D). We confirmed that BMDM generated from Sharpin ${ }^{-/ C} C y l{ }^{M-K O}$ mice were more

3 resistant to cell death than those from Sharpin-- mice and that the LysM-cre was effective in knocking

4 out CYLD in our BMDM cultures (Supplementary Figure S4E-G). We also crossed the Sharpin ${ }^{-/-}$strain $^{-1}$

5 to a $C x 3 c r l$-cre-driven conditional knockout of $C y l d\left(C y l d^{M P-K O}\right)$, which targets mononuclear

6 phagocytes including macrophages (61). The Cx3crl-cre-mediated deletion of $C y l d$ recapitulated the

7 effect observed earlier with LysM-cre (Supplementary Figure S5A-G). Results from the use of two

8 different cre strains that can delete Cyld in macrophages led us to conclude that CYLD-dependent

9 macrophage cell death may be a major driver of the inflammatory response in the skin of the Sharpin ${ }^{-/-}$

10 mice. 


\section{Discussion}

This study now provides genetic data that could account for the Sharpin ${ }^{-/}$phenotype. We propose that in wild type cells, SHARPIN-dependent regulation of CYLD suppresses its function, allowing K63-linked ubiquitin chains on RIPK1 to be sustained thus preventing RIPK1 from becoming a death-signaling molecule (Figure 6E, left panel). Thus, SHARPIN-dependent inhibition of CYLD is an essential component of Checkpoint 1 in the TNF signaling pathway that keeps cell death at bay. In the absence of SHARPIN, there is a failure to suppress CYLD, which dismantles K63-linked ubiquitin chains from RIPK1 to initiate the RIPK1-dependent death cascade (Figure 6E, right panel). We note that there is reduced phosphorylation of serine 418 of CYLD in SHARPIN-deficient cells, which is known to be phosphorylated by IKK $\beta$ and $\operatorname{IKK} \varepsilon(43,57)$. Reduced CYLD phosphorylation could be due to

11 reduced IKK activity in SHARPIN-deficient cells (7-9). In addition, CYLD is recruited to the receptor 12 complex in a SPATA2 and RNF31/HOIP-dependent manner (44-48) and since Sharpin ${ }^{-/}$cells have

13 reduced RNF31/HOIP level (7-9), this could reduce CYLD recruitment to the receptor complex and 14 subsequent phosphorylation by IKK. In light of published studies that phosphorylation of CYLD at a 15 cluster of serines around residue 418 inhibits its enzymatic activity $(43,57)$, it is likely that the defective

16 phosphorylation of CYLD in the SHARPIN-deficient cells enhanced CYLD's removal of ubiquitin

17 chains from RIPK1. Alternatively, phosphorylation could regulate CYLD function in a non-enzymatic

18 manner such as regulating its localization or its interaction with signaling partners. Furthermore, it is

19 possible that SHARPIN could inhibit CYLD through some other mechanism independent of

20 phosphorylation and this will be further explored.

In addition to phosphorylation, CYLD can also be negatively regulated by proteolysis mediated by CASP8 and MALT1 $(53,56)$. Interestingly, SHARPIN-deficient splenocytes may circumvent the

23 defect in CYLD phosphorylation by upregulating these proteolytic events suggesting that these

24 inhibitory mechanisms could compensate for each other. Furthermore, CYLD can be phosphorylated by 
multiple members of the IKK family including IKK $\beta$, IKK $\varepsilon$ and TBK1 suggesting that there may be redundancy in regulating CYLD phosphorylation in different cell types. This redundancy could explain the modest defect in CYLD phosphorylation in the Sharpin ${ }^{-/}$BMDM as macrophages express IKK $\varepsilon$ and TBK1 (62). Besides CYLD, these IKK kinases also directly phosphorylate RIPK1 to inhibit the deathsignaling function of RIPK1 (26-30). Taken together, the emerging picture is that a fully functional Checkpoint 1 needed to suppress ripoptocide is dependent on multiple post-translational modification of 7 several molecules.

Biologically, these results really point towards suppression of CYLD as central to immune homeostasis as perturbation in CYLD regulation, as observed in the Sharpin $^{-/}$mice, leads to multi-organ

10 inflammation and disruption of lymphoid tissues. It also demonstrates that CYLD functions as a pro-

11 inflammatory molecule since its deletion resolves the inflammation seen in the Sharpin ${ }^{-/-}$mice. These

12 aspects of CYLD in the proper functioning of the immune system have not been appreciated to date.

13 Previous studies with CYLD have described it as having an anti-inflammatory function (63-65),

14 primarily because of its inhibitory effect on the NF-kB pathway. We now show that CYLD can be

15 highly pro-inflammatory via its death-inducing function, which is revealed upon the loss of an upstream

16 inhibitory mechanism (e.g., SHARPIN/LUBAC). This is a significant advance in our understanding of

17 the role played by ubiquitin-modifiers in regulating immune homeostasis and response. Our studies

18 strongly suggest that the defects observed in human patients with mutations in $R N F 31 / H O I P$ and

$19 R B C K 1 / H O I L 1(17,18)$ could be due to inappropriate CYLD activity.

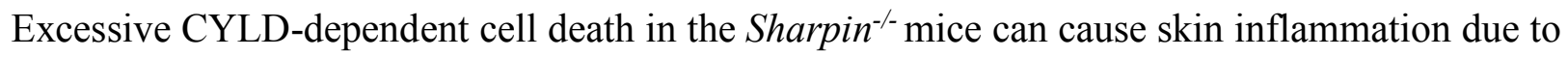

21 (1) death of epithelial cells that could result in cellular erosion and ulcers in and of itself, (2) loss of

22 barrier function in the epithelial layer and subsequent breach by commensal microbiota and

23 environmental contaminants (bedding and food), and/or (3) excessive cellular debris from dying cells

24 that are not properly cleared by phagocytes. The reversal of skin inflammation in Sharpin $^{-/}$mice 
1 following deletion of Fadd or Tradd in keratinocytes indicates that cell death in this cellular compartment is critical for inflammation (22). Our observation that the skin inflammation was also reduced by LysM-cre- and Cx3crl-cre-mediated deletion of Cyld suggested that cell death of macrophages also has a pro-inflammatory function. One explanation is that death of Sharpin ${ }^{-/}$epithelial cells is the initiating factor for the skin inflammation while death in macrophages plays an accessory role. SHARPIN-deficient macrophages can contribute to the skin inflammation by undergoing more death in response to invading microbiota and thereby amplifying the inflammatory response. Alternatively, because SHARPIN-deficient macrophages are more sensitive to death, they may be less effective in clearing up debris from dying epithelial cells. Since both LysM-cre- and Cx3crl-cre-

10 mediated deletion of $C y l d$ had the same effect, our data strongly suggest that improper cell death in

11 macrophages is highly inflammatory in the skin but does not have a role in other immune dysfunction in 12 the Sharpin $^{-/}$mice such as the disruption in splenic architecture.

14 survival to cell death by virtue of a genetic defect in CYLD regulation. The question remains as to the physiological function of this molecular switch in a normal individual and the role of TNF-mediated cell

16 death. Indeed, this question has remained unresolved for over forty years despite the fact that this was

17 the first cellular response attributed to TNF, as reflected in the name of this cytokine (66). This

18 conserved cell-killing function of TNF has been postulated to serve an evolutionary role against

19 microbial infection $(38,67)$. The analysis of the Sharpin $^{--}$mice suggests that a biological consequence

20 of TNF/CYLD-mediated ripoptocide is inflammation. In particular, the invocation of this death response

21 in macrophages is highly inflammatory since blocking this from occurring in macrophages ameliorated

22 the skin inflammation of the Sharpin ${ }^{-/}$mice. Therefore, it is probable that the inflammation caused by

23 ripoptocide has an anti-microbial function, as previously postulated (67). Since CYLD's death-inducing

24 function can be controlled by post-translational modifications, the speculation is that this early 
1 transcription-independent molecular switch may be disrupted by pathogens, thus providing a facile and rapid switch to cell death to generate an inflammatory response beneficial to the host. The IKK complex that phosphorylates CYLD is also necessary for induction of NF- $\kappa$ B-dependent inflammatory genes. Therefore, pathogens may encode inhibitors to block IKK as a strategy to limit the host expression of inflammatory genes and in so doing would also impair CYLD phosphorylation. This would be expected to unleash CYLD-dependent ripoptocide and the ensuing inflammation would function as a countermeasure against blockade of inflammatory gene induction by pathogens. It was particularly interesting that blocking CYLD-dependent ripoptocide in macrophages had a strong anti-inflammatory

9 effect on the dermatitis of the Sharpin ${ }^{-/}$mice. These sentinel cells are the major producers of

10 inflammatory cytokines in response to infection and are also often the host for replicative parasitic

11 pathogens. Therefore, the presence of this CYLD-regulated 'trap door' in macrophages would be

12 advantageous to the host. An example of this is infection by the bacteria pathogen Yersinia. It encodes

13 an effector molecule YopJ to block cytokine gene synthesis, which likely leads to the disruption of

14 Checkpoint 1 to trigger ripoptocide and this has been shown to be beneficial to the host $(29,68)$. It is

15 likely that there are other pathogens that behave similarly.

In summary, we have uncovered the mechanistic basis for the immune dysfunction observed in

17 linear ubiquitin-deficient mice. This involves a defect in regulating CYLD, a molecular switch that is

18 normally turned off to prevent TNF from inducing death. The finding that linear ubiquitin regulates the

19 function of CYLD, an enzyme that dismantles K63-linked ubiquitin chains, reveals positive

20 cooperativity between the two forms of ubiquitin modifications in regulating a cellular response. In

21 addition to providing an explanation for the immune system abnormalities caused by genetic defects in

22 linear ubiquitination, this molecular insight significantly advances our understanding of how

23 inflammation and immunodeficiency may be regulated by cell death. 


\section{Materials \& Methods}

2

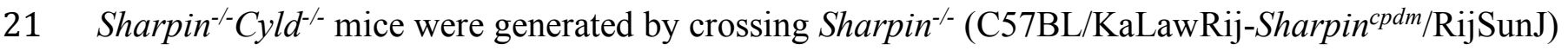

22 provided by Dr. John Sundberg with $C y l d^{--}$mice $\left(\mathrm{B} 6 ; 129 \mathrm{~S}-C y l d^{\operatorname{tm} 1 S c s}\right)$ provided by Dr. Shao-Cong Sun 
1 (69). Experiments were conducted using Sharpin ${ }^{+/+}$, Sharpin $^{-/-}$and Cyld ${ }^{-/}$littermates as controls.

2 Sharpin $^{--} C y l d^{M-K O}$ mice were generated by crossing Sharpin ${ }^{-/}$strain with the Cyldfloxflox (B6.129S-

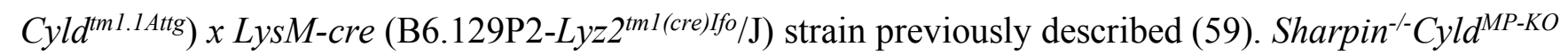

4 mice were generated by crossing Sharpin $^{-/}$strain with the Cyld floxflox (B6.129S-Cyld ${ }^{\text {tm 1.1Attg }}$ ) $x$ Cx3crl-

5 cre $\left(\mathrm{B} 6 \mathrm{~J} . \mathrm{B} 6 \mathrm{~N}(\mathrm{Cg}) C x 3 c r 1 c r e^{t m 1 .(c r e) J u n} / \mathrm{J}\right)$ strain. The Cx3cr1-cre was provided by Dr. Sergio Lira. All

6 experiments involving the use of animals were performed in agreement with approved protocols by the

7 Institutional Animal Care and Use Committee (IACUC) at the Icahn School of Medicine at Mount Sinai.

\section{Histology}

9 Skins, spleens, colons and small intestines were removed, fixed in 10\% neutral-buffered formalin, and

10 then transferred into $70 \%$ ethanol. Paraffin embedding, tissue sectioning, H\&E staining and

11 immunohistochemistry were performed by the Comparative Pathology Lab in the Center for

12 Comparative Medicine and Surgery, or by the Biorepository and Pathology CoRE at the Icahn School of

13 Medicine at Mount Sinai. Immunofluoresence labeling of skin sections was carried using the protocol

14 previously described (70).

\section{Clinical scoring}

16 The gross scoring scheme was modified from a system developed for a different mouse disease,

17 ulcerative dermatitis that occurs in C57BL/6 substrains (71). The clinical score combines gross

18 evaluation with histologic scoring from skin sections. In brief, 4 criteria were evaluated: character of

19 lesion $(0=$ none, $1=$ alopecia or excoriations only, or 1 small punctuate crust, $2=$ multiple, small punctuate

20 crusts or coalescing crusts $(>2 \mathrm{~mm})$, regions affected (head/cervical, thoracic, and/or abdominal/caudal;

$210=$ none, $1=<25 \%, 2=25-50 \%, 3=>50 \%$ ). The "regions affected" score in Hampton et al (71) was based

22 on the progression of the disease. Ulcerative dermatitis in B6 mice often started in the

23 intercapsular/dorsal back area (region 2) and progressed, thus encroachment on the head and face 
1 (region 1) was considered to be a severe score. In this study, the lesions often start in the ventral

2 chin/neck region (region 1) and progress to the thorax, abdomen and back. The scoring system was

3 modified to increase in severity based on the number of regions affected rather than the specific region

4 affected because of this difference in progression of skin disease. For the histologic lesions, scoring was

5 a subjective $0=$ none, $1=$ mild, $2=$ moderate, $3=$ marked scale. The scoring of inflammation in the

6 dermatitis was done using the following scale $0=$ none, $1=$ mild (focal or few foci of inflammation in

7 superficial dermis), $2=$ moderate (multiple foci with inflammation in deep dermis, with or without

8 ulcerations), and $3=$ severe (regionally extensive with inflammation extending to the subcutis and

9 ulceration).

10 Serum immunoglobulins, cytokines and chemokines assessment

11 Blood was collected via facial vein from 10-16 weeks old mice and spun at $1000 \mathrm{x} g$ for 10 min at room

12 temperature. Serum was then collected and stored at $-80^{\circ} \mathrm{C}$. $\operatorname{IgA}, \operatorname{IgE}, \operatorname{IgG}$ and $\operatorname{IgM}$ in the serum were

13 quantified using multiplex Luminex ${ }^{\circledR}$ Immunoglobulin Isotyping. IFNG, IL2, IL5, IL6, IL12/23p40,

14 eotaxin and CCL3 (MIP-1 $\alpha)$ in the serum were quantified using Luminex ${ }^{\circledR}$ Cytokines and Chemokines

15 Multiplex Assays.

\section{Stool IgA assessment}

17 Stool pellets obtained from 10-16 week old mice were placed in chilled PBS containing protease

18 inhibitors $(100 \mathrm{mg} / \mathrm{ml})$, repeatedly smashed and vortexed for 4-5 times. The suspension was then

19 centrifuged at $8000 \mathrm{xg}$ for $10 \mathrm{~min}$ at $4{ }^{\circ} \mathrm{C}$. The supernatants were collected and frozen at $-80^{\circ} \mathrm{C}$. IgA

20 levels were quantified using the Mouse IgA ELISA Quantitation Set (Bethyl Laboratories).

21 Primary adult dermal fibroblast (ADF) cultures 
1 A small section ( $2 \mathrm{~mm} \times 3 \mathrm{~mm}$ ) of the ears from adult mice were snipped, soaked in $70 \%$ ethanol for 30 sec and washed with sterile PBS. Tissues were then diced in culture media (DMEM with 10\% FBS and antibiotics) and spun down. The pellets were trypsinized for an hour at $37^{\circ} \mathrm{C}$ with intermittent hard vortexing prior to centrifugation. The pellets were then plated onto a tissue culture dish and cultured in

5 DMEM with $10 \%$ FBS and antibiotics at $37^{\circ} \mathrm{C}$ with $5 \% \mathrm{CO}_{2}$ for $1-2$ weeks.

\section{Total splenocytes and B cell isolation}

7 Spleens from 8-12-week-old adult mice were mashed and filtered through $70 \mu \mathrm{M}$ sterile funnel and with $\mathrm{RBC}$ lysis buffer for $5 \mathrm{~min}$. Total splenocytes were then rested at $37^{\circ} \mathrm{C}$ in a $5 \% \mathrm{CO}_{2}$ incubator for at

9 least $2 \mathrm{~h}$ before use. For B cell preparation, total splenocytes were subjected to negative selection using

10 BioLegend MojoSort Magnetic Cell Separation kit to obtain purified B cells. The purity was determined 11 by labeling for CD19 or CD40 by flow cytometry ( $>90 \%)$. B cells were rested for at least $2 \mathrm{~h}$ before use.

\section{Bone marrow-derived macrophage cultures}

13 Bone marrow-derived macrophages (BMDM) were generated by culturing bone marrow progenitors

14 from femurs and tibias of mice in RPMI containing 10\% FBS and 30\% L929 conditioned medium as

15 described (72). Day 8 to 11 cultures were used for all experiments.

\section{Retroviral transduction of MEF}

17 HEK293 EBNA cells were transfected by calcium phosphate precipitation with plasmids encoding

18 VSV-G and GAG-POL, together with an MMLV-based retroviral expression construct encoding control

19 or relevant genes. $48 \mathrm{~h}$ post-transfection, the viral supernatants were collected and used to infect MEF

20 by spinoculation. MEF were selected with puromycin or blasticidin 24 h subsequently. Sharpin ${ }^{-/-}$MEF

21 were complemented with a negative control or wild type mouse Sharpin gene. Both lines were

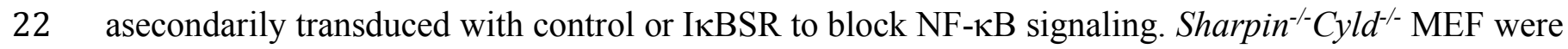


1 complemented with (i) control gene, (ii) mouse Sharpin wild type alone, (iii) mouse Cyld wild type

2 alone, or (iv) both mouse Sharpin and Cyld wild type alleles. Cyld ${ }^{-/-}$MEF were complemented with (i)

3 control gene, (ii) mouse $C y l d$ wild type allele, or (iii) mouse $C y l d^{S 7 A}$ mutant allele. The $C y l d^{S 7 A}$ ser-to-ala

4 mutations were generated using Quikchange Multi Site-Directed Mutagenesis Kit (Agilent).

\section{CYLD knockdown in MEF}

6 HEK 293 EBNA cells were transfected by calcium phosphate precipitation with plasmids encoding

$7 \quad$ VSV-G and GAG-POL, together with Mission ${ }^{\circledR}$ shRNA lentiviral vectors targeting CYLD or a non-

8 targeting shRNA. Lentiviral infection of Sharpin $^{-/}$MEF was carried out as described above for

9 retroviral transduction.

\section{Cell death assays}

11 Cellular death analyses were performed by Annexin V or propidium iodide staining and flow cytometry

12 as previously described (53). Cell death was also quantified using the CellTiter-Glo Luminescent Cell

13 Viability Assay (Promega). Biological triplicates were used in the experiments for ADF and BMDM cell

14 death analysis.

\section{Co-immunoprecipitation}

16 For FADD immunoprecipitation, $2 \times 10^{6}$ cells ADF and MEF or 3-4 x $10^{6}$ BMDM per sample were

17 stimulated and then lysed in buffer containing $20 \mathrm{mM}$ Tris $\mathrm{pH} 7.4,150 \mathrm{mM}$ sodium chloride, $10 \%$

18 glycerol, $0.2 \%$ NP-40, $0.1 \mathrm{mM}$ sodium orthovandate, $5 \mathrm{mM} \beta$-glycerophosphate and Protease Inhibitor

19 Cocktail Set V for $20 \mathrm{~min}$ on ice. Lysates were cleared by centrifugation at $10,000 \mathrm{xg}$ at $4^{\circ} \mathrm{C}$ and

20 protein concentration measured using Pierce BCA (Thermo Fisher). Equivalent amount of protein in

21 each sample were immunoprecipitated by rotating with $0.25-1 \mu \mathrm{g}$ of FADD antibody (M19) overnight at

$224^{\circ} \mathrm{C}$. Immune complexes were precipitated with Protein A/G beads. After extensive washing, the beads 
1 were eluted with SDS-sample buffer at $70^{\circ} \mathrm{C}$ for $20 \mathrm{~min}$. Blotting with anti-RIPK1 was carried out

2 subsequently.

3 For RIPK1 immunoprecipitation, $0.84 \times 10^{6} \mathrm{MEF}$ stimulated and then lysed in buffer containing $20 \mathrm{mM}$

4 Tris $\mathrm{pH}$ 7.4, $150 \mathrm{mM}$ sodium chloride, 10\% glycerol, 1\% TritonX-100, 0.5mM DTT, 10mM N-

5 Ethylmaleimide, $0.1 \mathrm{mM}$ sodium orthovandate, $5 \mathrm{mM} \beta$-glycerophosphate and Protease Inhibitor

6 Cocktail Set V for 20 min on ice. Lysates were cleared by centrifugation at $10,000 \times g$ at $4^{\circ} \mathrm{C}$. One tenth

7 of the lysate was set aside for SDS-PAGE and sequential blotting with anti-RIPK1 and anti-ACTIN. The

8 reminder of the lysates was immunoprecipitated by rotating with $0.4 \mu \mathrm{g}$ of RIPK 1 antibody overnight at

$9 \quad 4^{\circ} \mathrm{C}$. Immune complexes were precipitated with Protein $\mathrm{A} / \mathrm{G}$ beads. After extensive washing, the beads

10 were eluted with SDS-sample buffer at $70^{\circ} \mathrm{C}$ for $20 \mathrm{~min}$. Blotting with anti-RIPK1 was carried out

11 subsequently.

\section{Western blot Analysis}

13 Cellular lysates obtained using 1\% Triton X-100 were resolved by reducing SDS PAGE. For P-MLKL,

14 lysates were generated using M2 buffer as previously described (73). Blotting was conducted using

15 standard techniques and detected by LiCor Odyssey or by chemiluminescence. Semi-quantification of

16 the blots was carried using Image J software (74).

\section{Statistical Analysis}

18 Statistics were performed using one-way Anova, Student's t-test or Mann Whitney U test. (*p<0.05,

$19 * * \mathrm{k}<0.01, * * * \mathrm{p}<0.001)$ 


\section{Author contributions}

2 R.L.A designed and performed the experiments. J.P.S. and S.C.C. provided Sharpin ${ }^{-/-}$and Cyld ${ }^{-/}$mice,

3 respectively. V.G. did the clinical scoring in the double-blind study. J.P.S. reviewed the histopathology.

4 H.X. and S.A.L. provided technical guidance. P.S.H. provided critical comments, edited the manuscript

5 and provided financial support through fellowship grants. R.L.A. and A.T.T. wrote the manuscript and

6 directed the studies.

\section{Acknowledgements}

9 We would like to thank the following from the Icahn School of Medicine at Mount Sinai: Mr. Alan Soto

10 from Biorepository and Pathology CoRE services for processing the histology, Ms. Ying Dai from the

11 Comparative Pathology Laboratory in the Center for Comparative Medicine and Surgery for imaging,

12 and Drs. Thomas Krauss and Thomas Moran for the use of the Luminex instrument. This work was

13 supported by National Institutes of Health (NIH) grants AI052417 (A.T.T.), AI104521 (A.T.T.),

14 AI132405 (A.T.T \& P.S.H.), DK072201 (S.A.L. \& A.T.T.), CA161373 (S.A.L.), AI064639 (S.-C. S)

15 and AR049288 (J.P.S.). This work was also supported by a Senior Research Award \#253097 (A.T.T.)

16 and \#330239 (S.A.L.) from the Crohn's and Colitis Foundation of America. R.L.A was supported by

17 National Institute of Health training grants: AI078892, GM062754, and A1007605. We declare that

18 there are no financial conflicts of interest. 


\section{References}

1. Y. Shimizu, L. Taraborrelli, H. Walczak, Linear ubiquitination in immunity. Immunol Rev 266, 190-207 (2015).

2. K. Iwai, H. Fujita, Y. Sasaki, Linear ubiquitin chains: NF-kappaB signalling, cell death and beyond. Nat Rev Mol Cell Biol 15, 503-508 (2014).

3. H. Walczak, K. Iwai, I. Dikic, Generation and physiological roles of linear ubiquitin chains. BMC Biol 10, 23 (2012).

4. T. Kirisako et al., A ubiquitin ligase complex assembles linear polyubiquitin chains. EMBO J 25, 4877-4887 (2006).

5. F. Tokunaga et al., Involvement of linear polyubiquitylation of NEMO in NF-kappaB activation. Nat Cell Biol 11, 123-132 (2009).

6. T. L. Haas et al., Recruitment of the linear ubiquitin chain assembly complex stabilizes the TNF-R1 signaling complex and is required for TNF-mediated gene induction. Mol Cell 36, 831-844 (2009).

7. F. Tokunaga et al., SHARPIN is a component of the NF-kappaB-activating linear ubiquitin chain assembly complex. Nature 471, 633-636 (2011).

8. F. Ikeda et al., SHARPIN forms a linear ubiquitin ligase complex regulating NF-kappaB activity and apoptosis. Nature 471, 637-641 (2011).

9. B. Gerlach et al., Linear ubiquitination prevents inflammation and regulates immune signalling. Nature 471, 591-596 (2011).

10. N. Peltzer et al., LUBAC is essential for embryogenesis by preventing cell death and enabling haematopoiesis. Nature 557, 112-117 (2018).

11. F. Ikeda, Linear ubiquitination signals in adaptive immune responses. Immunol Rev 266, 222-236 (2015).

12. S. Rahighi et al., Specific recognition of linear ubiquitin chains by NEMO is important for NF-kappaB activation. Cell 136, 1098-1109 (2009).

13. H. Fujita et al., Mechanism underlying IkappaB kinase activation mediated by the linear ubiquitin chain assembly complex. Mol Cell Biol 34, 1322-1335 (2014).

14. V. Anest et al., A nucleosomal function for IkappaB kinase-alpha in NF-kappaB-dependent gene expression. Nature 423, 659-663 (2003).

15. Y. Yamamoto, U. N. Verma, S. Prajapati, Y. T. Kwak, R. B. Gaynor, Histone H3 phosphorylation by IKK-alpha is critical for cytokine-induced gene expression. Nature 423, 655659 (2003).

16. J. Yan et al., Inactivation of BAD by IKK inhibits TNFalpha-induced apoptosis independently of NF-kappaB activation. Cell 152, 304-315 (2013).

17. B. Boisson et al., Immunodeficiency, autoinflammation and amylopectinosis in humans with inherited HOIL-1 and LUBAC deficiency. Nat Immunol 13, 1178-1186 (2012).

18. B. Boisson et al., Human HOIP and LUBAC deficiency underlies autoinflammation, immunodeficiency, amylopectinosis, and lymphangiectasia. J Exp Med 212, 939-951 (2015). 19. H. HogenEsch et al., A spontaneous mutation characterized by chronic proliferative dermatitis in C57BL mice. Am J Pathol 143, 972-982 (1993).

20. H. HogenEsch, S. Janke, D. Boggess, J. P. Sundberg, Absence of Peyer's patches and abnormal lymphoid architecture in chronic proliferative dermatitis (cpdm/cpdm) mice. J Immunol 162, 3890-3896 (1999).

21. R. E. Seymour et al., Spontaneous mutations in the mouse Sharpin gene result in multiorgan inflammation, immune system dysregulation and dermatitis. Genes Immun 8, 416-421 (2007). 
22. S. Kumari et al., Sharpin prevents skin inflammation by inhibiting TNFR1-induced keratinocyte apoptosis. Elife 3 (2014).

23. J. A. Rickard et al., TNFR1-dependent cell death drives inflammation in Sharpin-deficient mice. Elife 3 (2014).

24. S. B. Berger et al., Cutting Edge: RIP1 kinase activity is dispensable for normal development but is a key regulator of inflammation in SHARPIN-deficient mice. J Immunol 192, 5476-5480 (2014).

25. R. L. Ang, M. Chan, A. T. Ting, Ripoptocide - A Spark for Inflammation. Front Cell Dev Biol 7, 163 (2019).

26. D. Xu et al., TBK1 Suppresses RIPK1-Driven Apoptosis and Inflammation during Development and in Aging. Cell 174, 1477-1491 e1419 (2018).

27. E. Lafont et al., TBK1 and IKKepsilon prevent TNF-induced cell death by RIPK1 phosphorylation. Nat Cell Biol 20,1389-1399 (2018).

28. Y. Dondelinger et al., NF-kappaB-Independent Role of IKKalpha/IKKbeta in Preventing RIPK1 Kinase-Dependent Apoptotic and Necroptotic Cell Death during TNF Signaling. Mol Cell 60, 63-76 (2015).

29. Y. Dondelinger et al., Serine 25 phosphorylation inhibits RIPK1 kinase-dependent cell death in models of infection and inflammation. Nat Commun 10,1729 (2019).

30. C. Koppe et al., IkappaB kinasealpha/beta control biliary homeostasis and hepatocarcinogenesis in mice by phosphorylating the cell-death mediator receptor-interacting protein kinase 1. Hepatology 64, 1217-1231 (2016).

31. S. J. Justus, A. T. Ting, Cloaked in ubiquitin, a killer hides in plain sight: the molecular regulation of RIPK1. Immunol Rev 266, 145-160 (2015).

32. X. Wu, M. Karin, Emerging roles of Lys63-linked polyubiquitylation in immune responses. Immunol Rev 266, 161-174 (2015).

33. M. A. O'Donnell, D. Legarda-Addison, P. Skountzos, W. C. Yeh, A. T. Ting, Ubiquitination of RIP1 regulates an NF-kappaB-independent cell-death switch in TNF signaling. Curr Biol 17, 418424 (2007).

34. L. Wang, F. Du, X. Wang, TNF-alpha induces two distinct caspase-8 activation pathways. Cell 133, 693-703 (2008).

35. M. J. Bertrand et al., cIAP1 and cIAP2 Facilitate Cancer Cell Survival by Functioning as E3 Ligases that Promote RIP1 Ubiquitination. Mol Cell 30, 689-700 (2008).

36. M. A. O'Donnell, A. T. Ting, Chronicles of a death foretold: dual sequential cell death checkpoints in TNF signaling. Cell Cycle 9, 1065-1071 (2010).

37. M. A. O'Donnell, A. T. Ting, RIP1 comes back to life as a cell death regulator in TNFR1 signaling. FEBS J 278, 877-887 (2011).

38. A. T. Ting, M. J. Bertrand, More to Life than NF-kappaB in TNFR1 Signaling. Trends Immunol 37, 535-545 (2016).

39. S. He et al., Receptor interacting protein kinase-3 determines cellular necrotic response to TNF-alpha. Cell 137, 1100-1111 (2009).

40. J. E. Vince et al., IAP antagonists target cIAP1 to induce TNFalpha-dependent apoptosis. Cell 131, 682-693 (2007).

41. S. L. Petersen et al., Autocrine TNFalpha signaling renders human cancer cells susceptible to Smac-mimetic-induced apoptosis. Cancer Cell 12, 445-456 (2007).

42. S. C. Sun, CYLD: a tumor suppressor deubiquitinase regulating NF-kappaB activation and diverse biological processes. Cell Death Differ 17, 25-34 (2010).

43. W. Reiley, M. Zhang, X. Wu, E. Granger, S. C. Sun, Regulation of the deubiquitinating enzyme 48 CYLD by IkappaB kinase gamma-dependent phosphorylation. Mol Cell Biol 25, 3886-3895 (2005). 
44. T. Takiuchi et al., Suppression of LUBAC-mediated linear ubiquitination by a specific interaction between LUBAC and the deubiquitinases CYLD and OTULIN. Genes Cells 19, 254-272 (2014).

45. P. R. Elliott et al., SPATA2 Links CYLD to LUBAC, Activates CYLD, and Controls LUBAC Signaling. Mol Cell 63, 990-1005 (2016).

46. S. Kupka et al., SPATA2-Mediated Binding of CYLD to HOIP Enables CYLD Recruitment to Signaling Complexes. Cell Rep 16, 2271-2280 (2016).

47. L. Schlicher et al., SPATA2 promotes CYLD activity and regulates TNF-induced NF-kappaB signaling and cell death. EMBO Rep 17, 1485-1497 (2016).

48. S. A. Wagner, S. Satpathy, P. Beli, C. Choudhary, SPATA2 links CYLD to the TNF-alpha receptor signaling complex and modulates the receptor signaling outcomes. EMBO J 35, 18681884 (2016).

49. P. Draber et al., LUBAC-Recruited CYLD and A20 Regulate Gene Activation and Cell Death by Exerting Opposing Effects on Linear Ubiquitin in Signaling Complexes. Cell Rep 13, 2258-2272 (2015).

50. A. Wright et al., Regulation of early wave of germ cell apoptosis and spermatogenesis by deubiquitinating enzyme CYLD. Dev Cell 13, 705-716 (2007).

51. Y. Dondelinger et al., RIPK3 contributes to TNFR1-mediated RIPK1 kinase-dependent apoptosis in conditions of cIAP1/2 depletion or TAK1 kinase inhibition. Cell Death Differ 20, 13811392 (2013).

52. N. Vanlangenakker et al., cIAP1 and TAK1 protect cells from TNF-induced necrosis by preventing RIP1/RIP3-dependent reactive oxygen species production. Cell Death Differ 18, 656665 (2011).

53. M. A. O'Donnell et al., Caspase 8 inhibits programmed necrosis by processing CYLD. Nat Cell Biol 13, 1437-1442 (2011).

54. R. Seymour, B. J. Shirley, H. Hogenesch, L. D. Shultz, J. P. Sundberg, Loss of function of the mouse Sharpin gene results in Peyer's patch regression. PLoS One 8, e55224 (2013).

55. O. Micheau, J. Tschopp, Induction of TNF receptor I-mediated apoptosis via two sequential signaling complexes. Cell 114, 181-190 (2003).

56. J. Staal et al., T-cell receptor-induced JNK activation requires proteolytic inactivation of CYLD by MALT1. EMBO J 30, 1742-1752 (2011).

57. J. E. Hutti et al., Phosphorylation of the tumor suppressor CYLD by the breast cancer oncogene IKKepsilon promotes cell transformation. Mol Cell 34, 461-472 (2009).

58. L. R. Klei et al., MALT1 Protease Activation Triggers Acute Disruption of Endothelial Barrier Integrity via CYLD Cleavage. Cell Rep 17, 221-232 (2016).

59. D. Legarda et al., CYLD Proteolysis Protects Macrophages from TNF-Mediated Autonecroptosis Induced by LPS and Licensed by Type I IFN. Cell Rep 15, 2449-2461 (2016).

60. C. S. Potter et al., Chronic proliferative dermatitis in Sharpin null mice: development of an autoinflammatory disease in the absence of B and T lymphocytes and IL4/IL13 signaling. PLoS One 9, e85666 (2014).

61. S. Yona et al., Fate mapping reveals origins and dynamics of monocytes and tissue macrophages under homeostasis. Immunity 38, 79-91 (2013).

62. A. K. Perry, E. K. Chow, J. B. Goodnough, W. C. Yeh, G. Cheng, Differential requirement for TANK-binding kinase-1 in type I interferon responses to toll-like receptor activation and viral infection. J Exp Med 199, 1651-1658 (2004).

63. J. H. Lim et al., Tumor suppressor CYLD acts as a negative regulator for non-typeable Haemophilus influenza-induced inflammation in the middle ear and lung of mice. PLoS One 2, e1032 (2007). 
1 64. R. Massoumi, K. Chmielarska, K. Hennecke, A. Pfeifer, R. Fassler, Cyld inhibits tumor cell 2 proliferation by blocking Bcl-3-dependent NF-kappaB signaling. Cell 125, 665-677 (2006).

3 65. J. Zhang et al., Impaired regulation of NF-kappaB and increased susceptibility to colitis4 associated tumorigenesis in CYLD-deficient mice. J Clin Invest 116, 3042-3049 (2006).

5 66. E. A. Carswell et al., An endotoxin-induced serum factor that causes necrosis of tumors. 6 Proc Natl Acad Sci US A 72, 3666-3670 (1975).

7 67. L. J. Old, Tumor necrosis factor (TNF). Science 230, 630-632 (1985).

8 68. L. W. Peterson et al., RIPK1-dependent apoptosis bypasses pathogen blockade of innate 9 signaling to promote immune defense. J Exp Med 214, 3171-3182 (2017).

10 69. W. W. Reiley et al., Regulation of T cell development by the deubiquitinating enzyme CYLD. 11 Nat Immunol 7, 411-417 (2006).

12 70. G. Bongers et al., Interplay of host microbiota, genetic perturbations, and inflammation 13 promotes local development of intestinal neoplasms in mice. J Exp Med 211, 457-472 (2014).

14 71. A. L. Hampton et al., Progression of ulcerative dermatitis lesions in C57BL/6Crl mice and 15 the development of a scoring system for dermatitis lesions. J Am Assoc Lab Anim Sci 51, 586-593 16 (2012).

17 72. X. Zhang, R. Goncalves, D. M. Mosser, The isolation and characterization of murine 18 macrophages. Curr Protoc Immunol Chapter 14, Unit 1411 (2008).

19 73. Z. Cai et al., Plasma membrane translocation of trimerized MLKL protein is required for 20 TNF-induced necroptosis. Nat Cell Biol 16, 55-65 (2014).

21 74. C. A. Schneider, W. S. Rasband, K. W. Eliceiri, NIH Image to ImageJ: 25 years of image

22 analysis. Nat Methods 9, 671-675 (2012). 


\section{Figure Legends}

2 Figure 1. Cyld is essential for the development of inflammation in Sharpin ${ }^{-/}$mice.

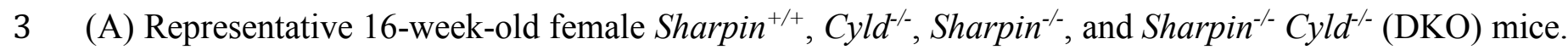

4 (B) A cohort of 16-week-old female mice was examined by a pathologist in a double-blind fashion. The

5 clinical score of mice from the indicated genotypes are shown ( $\mathrm{n}=4$ per genotype). One-way ANOVA

6 analysis was performed. $* \mathrm{P}<0.05, * * \mathrm{P}<0.01$.

7 (C) Histology of skin taken from mice in (B) by H\&E staining (100X). Representative

8 photomicrographs from each genotype are shown. E: epidermis; D: dermis; HF: hypodermal fat; PCM:

9 panniculus carnosus muscle; $\mathrm{U}$ : ulcerated area with complete loss of epidermis, replacement by necrosis

10 and inflammation; DI: dermal inflammation.

11 (D) Skin sections taken from mice in (B) were analyzed by immunohistochemistry with anti-CD45 to

12 label hematopoietic cells (100X). CD45+ cells are stained red. Representative photomicrographs from

13 each genotype are shown.

14 (E) Rectangular insert of the Sharpin ${ }^{-/}$skin section from panel (D) above is enlarged for visualization of 15 the CD45+ cells.

16 (F) Skin sections taken from mice in (B) were analyzed by immunofluorescence with anti-F4/80 to

17 detect macrophages (100X). Representative photomicrographs from each genotype are shown.

18 (g) Rectangular insert of the Sharpin ${ }^{-/}$skin section from panel (F) above is enlarged for visualization of $19 \mathrm{~F} 4 / 80+$ macrophages.

21 Figure 2. Cyld deletion reverses lymphoid tissue defects in Sharpin ${ }^{-/}$mice. 
1 (A) Gross analysis of spleens, mesenteric lymph nodes (MLN) and thymi obtained from 16-week-old mice. Spleens from two individual mice of each genotype are shown.

3 (B) Histology of spleens taken from mice of the indicated genotypes by H\&E staining (100X).

4 Representative photomicrographs from each genotype are shown.

5 (C) H\&E staining of Swiss rolls of small intestines obtained from 16 week-old mice of the indicated

6 genotypes. White arrows indicate Peyer's patches. Images of individual Peyer's patches are shown

7 below. Note Sharpin $^{-/-}$mice lack Peyer's patches.

8 (D) The number of Peyer's patches was enumerated in each mouse of the different genotypes. One-way

$9 \quad$ ANOVA analysis was performed. $* \mathrm{P}<0.05, * * \mathrm{P}<0.01$.

10 (E) Level of serum IgG, IgA, IgE and IgM from 10-16-week-old mice were measured by Luminex

11 Multiplex assays. Each data point is from an individual mouse ( $n=6-8$ mice per genotype). Error bars

12 represent mean $\pm \mathrm{SD}$ and Student's t-test was performed. $* \mathrm{P}<0.05, * * \mathrm{P}<0.01, * * * \mathrm{P}<0.001$.

13 (F) Stool IgA level in mice from the indicated genotypes were analyzed by ELISA. Each data point is

14 from an individual mouse ( $\mathrm{n}=6-8$ mice per genotype). Error bars represent mean $\pm \mathrm{SD}$ and Student's t-test

15 was performed. $* \mathrm{P}<0.05, * * \mathrm{P}<0.01, * * * \mathrm{P}<0.001$

16 (G) Level of serum IFNG, IL5 and IL12/23p40 from 10-16-week-old mice were measured by Luminex

17 Multiplex assays. Each data point is from an individual mouse ( $n=9-12$ mice per genotype). Error bars

18 represent mean $\pm \mathrm{SD}$ and Student's t-test was performed. $* \mathrm{P}<0.05, * * \mathrm{P}<0.01$. 
1 (A) Adult dermal fibroblasts (ADF) from Sharpin $^{+/+}$and Sharpin $^{-/}$mice were treated with mTNF (100 $\mathrm{ng} / \mathrm{ml}$ ) for the indicated minutes. CYLD phosphorylation was detected by blotting with anti-phospho-

3 CYLD (Ser 418), followed by blotting for total CYLD, SHARPIN and $\beta$-ACTIN.

4 (B) Sharpin + , Sharpin ${ }^{-/}$and Sharpin $^{-/}$MEF complemented with Sharpin or control gene were

5 stimulated with mTNF (100 ng/ml) for the indicated times. CYLD phosphorylation was analyzed as in 6 (A).

7 (C) Sharpin +- , and Sharpin ${ }^{-/-}$MEF were stimulated with $100 \mathrm{ng} / \mathrm{ml} \mathrm{mTNF}$ for $10 \mathrm{~min}$ in the presence of 80 to $50 \mu \mathrm{M}$ TPCA-1, a highly selective inhibitor against IKK $\beta$. CYLD phosphorylation was analyzed as 9 in (A).

10 (D) Purified B cells from Sharpin ${ }^{+/+}$and Sharpin $^{-/-}$mice were treated with mTNF (100 ng/ml) for the 11 indicated minutes. CYLD phosphorylation was analyzed as in (A).

12 (E) Total splenocytes from Sharpin ${ }^{+/+}$and Sharpin $^{-/}$mice were treated with mTNF (100 ng/ml) for the

13 indicated minutes. CYLD phosphorylation was analyzed as in (A). The indicated $25 \mathrm{kD}$ and $35 \mathrm{kD}$

14 fragments of CYLD correspond to N-terminal fragments from proteolysis by CASP8 and MALT1, 15 respectively. Data in panels A-E are representative of at least three independent experiments conducted 16 for each panel.

Figure 4. Sharpin ${ }^{--}$cells are more susceptible to CYLD-mediated cell death.

19 (A) Sharpin ${ }^{+/+}$, Sharpin $^{-/}, C y l d^{-/}$and DKO ADF were treated with mTNF (10 ng/ml) for $24 \mathrm{~h}$ in the 20 presence or absence of $\mathrm{zVAD}(20 \mu \mathrm{M})$ to induce necroptosis or cycloheximide $(\mathrm{CHX}, 1 \mu \mathrm{g} / \mathrm{ml})$ to

21 induce apoptosis. Cell death was analyzed by propidium iodide staining and flow cytometry. Data from

22 three independent experiments are shown. Error bars represent mean \pm SD and one-way ANOVA 
1 analysis was performed. ***P $<0.001$ Sharpin $^{-/-}$versus Sharpin ${ }^{+/+}$ADF. P value of DKO versus

2 Sharpin $^{+/+} \mathrm{ADF}$ is not significant.

3 (B) CYLD and SHARPIN expression in Sharpin $^{+/+}, C_{y l d}{ }^{-/}$, Sharpin $^{-/}$, and DKO ADF.

4 (C-D) Sharpin $^{+/+}$, Cyld ${ }^{-/}$, Sharpin $^{-/}$and DKO ADF were stimulated with mTNF (100 ng/ml) in the

5 presence of CHX $(1 \mu \mathrm{g} / \mathrm{ml})$ for 3 and $6 \mathrm{~h}$. Apoptosis was examined by blotting for cleaved CASP8 and

6 cleaved CASP3 (C) or cleaved PARP (D). For each panel, data shown are representative of at least three

$7 \quad$ independent experiments.

8 (E) Necroptosis was analyzed by blotting for phospho-MLKL (P-MLKL) in Sharpin ${ }^{+/+}$, Sharpin $^{-/}, C^{\prime} l^{-}$

9 /-, and DKO ADF stimulated with mTNF $(25 \mathrm{ng} / \mathrm{ml})$ in the presence of zVAD $(20 \mu \mathrm{M})$ for 1.5 and $3 \mathrm{~h}$.

10 Result shown is representative of at least three independent experiments.

11 (F) Sharpin ${ }^{+/+}$, Sharpin'-- Cyld ${ }^{-/}$, and DKO ADF were stimulated with mTNF (100 ng/ml) in the

12 presence of $\mathrm{CHX}(1 \mu \mathrm{g} / \mathrm{ml})$ and $\mathrm{zVAD}(20 \mu \mathrm{M})$ for 2 and $4 \mathrm{~h}$. The death-inducing signaling complex

13 (DISC) was isolated by FADD immunoprecipitation and sequentially blotted for RIPK1 and FADD.

14 Experiment was repeated twice with similar results.

15 (G) Residues 411 to 445 of wild type mouse CYLD encompassing the cluster of seven serines

16 equivalent to those reported to be phosphorylated on human CYLD, and the corresponding ser-to-ala

17 mutations in the non-phosphorylatable CYLD-S7A mutant are depicted. Ser417 of mouse CYLD is

18 equivalent to Ser418 of human CYLD. Cyld ${ }^{-/-}$MEF were transduced with retroviruses encoding a

19 control, wild type CYLD or CYLD-S7A. 24 h after transduction, cells were trypsinized and plated at a

20 density of $3.5 \times 10^{4}$ cells/well in 24 -well plates. The next day, cells were treated with DMSO as negative

21 control (UN), $100 \mathrm{ng} / \mathrm{ml} \mathrm{mTNF}(\mathrm{T}), 50 \mathrm{uM}$ zVAD-FMK (Z) or the combination of the two (TZ).

22 Propidium iodide staining and flow cytometry was performed 24 hours after stimulation. Numbers

23 indicate the percentage of PI+ cells. Aliquots of the retroviral transduced MEF were also analyzed by 
1 western blotting to demonstrate equivalent expression of wild type and mutant CYLD. Data shown is

2 from one of two independent replicate experiments with similar result.

4 Figure 5. Sharpin ${ }^{-/}$myeloid cells are more susceptible to CYLD-mediated cell death.

5 (A-C) Sharpin $^{+/+}$and Sharpin+-- bone marrow derived macrophages (BMDM) were stimulated with (A)

$6100 \mathrm{ng} / \mathrm{ml} \mathrm{mTNF}$, (B) $10 \mathrm{ng} / \mathrm{ml}$ LPS and (C) $2.5 \mu \mathrm{g} / \mathrm{ml}$ polyIC for the indicated times. CYLD

7 phosphorylation was analyzed as in Figure 3A. Blots shown are representative of three independent

8 experiments performed with each stimulus.

9 (D-E) Sharpin ${ }^{+/+}, S_{\text {Sharpin }}^{-/}, C y l d^{-/}$and DKO BMDM were stimulated with the indicated doses of (D)

10 LPS and (E) polyIC in the presence of DMSO or zVAD $(20 \mu \mathrm{M})$ for $24 \mathrm{~h}$. Cell death was analyzed as in

11 Figure 4A. Error bars represent mean $\pm \mathrm{SD}$ and one-way ANOVA analysis was performed. $* * * \mathrm{P}<0.001$

12 Sharpin $^{-/}$versus Sharpin ${ }^{+/+}$BMDM. P value of DKO versus Sharpin ${ }^{+/+}$BMDM is not significant.

13 (F) Sharpin ${ }^{+/+}$, Sharpin $^{-/}, C y l d^{-/}$and DKO BMDM were stimulated with mTNF (100 ng/ml) in the

14 presence of $\mathrm{CHX}(0.25 \mu \mathrm{g} / \mathrm{ml})$ and $\mathrm{zVAD}(20 \mu \mathrm{M})$ for 4 and $6 \mathrm{~h}$. Translocation of RIPK1 to the DISC

15 was analyzed as in Figure 4F. Result shown is representative of three independent experiments

16 performed.

17 (G) Skin sections from mice of the indicated genotypes were labeled by immunofluorescence with anti-

18 F4/80 and anti-cleaved CASP3 (CC3) to detect macrophages and dying cells, respectively.

19 Representative photomicrographs (100X) from each genotype are shown. White arrowheads indicate co-

20 localization of macrophages with cleaved CASP3.

21 (H) Rectangular insert of the Sharpin $^{-/}$skin section from panel (G) above is enlarged for visualization of 22 the F4/80 and cleaved CASP3 signals. 
2 Figure 6. CYLD-mediated death of myeloid cells is necessary to cause skin inflammation in

3 Sharpin $^{--}$mice.

4 (A) Photograph of 10-16-week-old Sharpin ${ }^{-/} C y l d^{M-K O}$ mice in comparison to a 9-week-old Sharpin ${ }^{-/-}$

5 mouse.

(B) H\&E staining of skin sections from 16-week-old Sharpin ${ }^{-/}$and Sharpin ${ }^{-/} C y l d^{M-K O}$ mice (100X). Top arrow: orthokeratotic hyperkeratosis and serocellular crust; middle arrow: thickened epidermis; bottom

8 arrow: thickened dermis.

9 (C) Gross analysis of spleens, mesenteric lymph nodes and thymi from 16-week-old Sharpin ${ }^{+/+}$,

10 Sharpin $^{-/}$and Sharpin ${ }^{-/} C y l d^{M-K O}$ mice.

11 (D) H\&E staining of spleen sections from 16-week-old Sharpin ${ }^{+/+}$and Sharpin ${ }^{-/} C y l{ }^{M-K O}$ mice.

12 (E) Model of CYLD suppression by linear ubiquitin. In wild type cells, the LUBAC-dependent IKK

13 phosphorylates CYLD to inactivate it. LUBAC could also suppress CYLD via a phosphorylation-

14 independent mechanism. These mechanisms suppress CYLD thereby keeping RIPK1 in a survival

15 mode. In addition, IKK can directly phosphorylate RIPK1 to inhibit its death-signaling function. Linear

16 ubiquitin cooperates positively with K63-linked ubiquitin to maintain cell survival by preventing the

17 dismantling of K63-linked ubiquitin chains. In SHARPIN-deficient cells, there is diminished

18 suppression of CYLD. Therefore, CYLD is active and removes K63-linked ubiquitin chains from

19 RIPK1. This enables RIPK1 to associate with the DISC to induce cell death leading to multi-organ

20 inflammation, abnormal lymphoid tissues and immunodeficiency. 
Figure blioRxiv preprint doi: https://doi.org/10.1101/2020.01.27.919076; this version posted January 27, 2020. The copyright holder for this preprint (which was not certified by peer review) is the author/funder. All rights reserved. No reuse allowed without permission.

(A)

Dorsal:

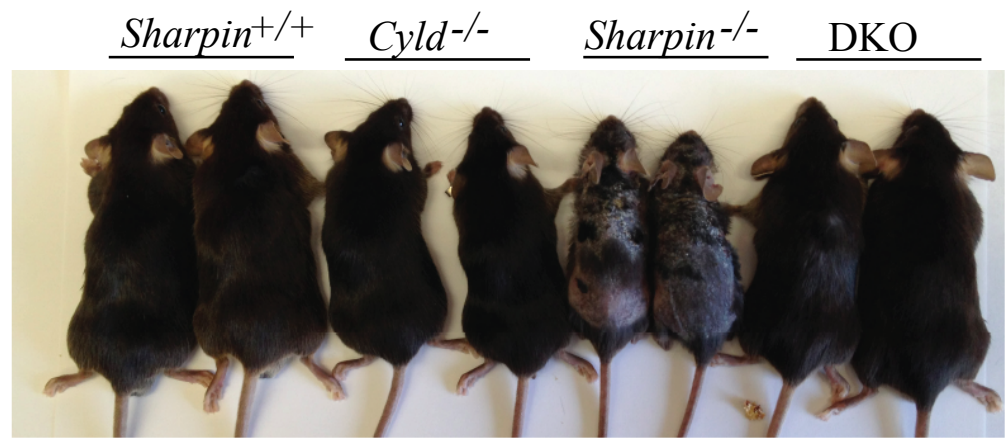

Ventral:

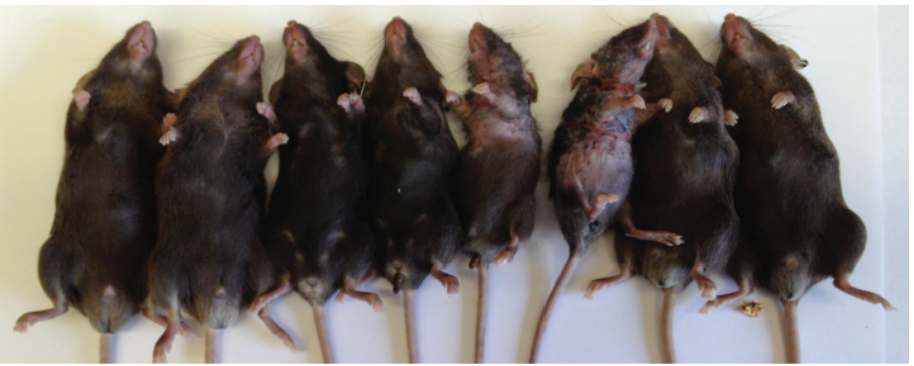

(B)

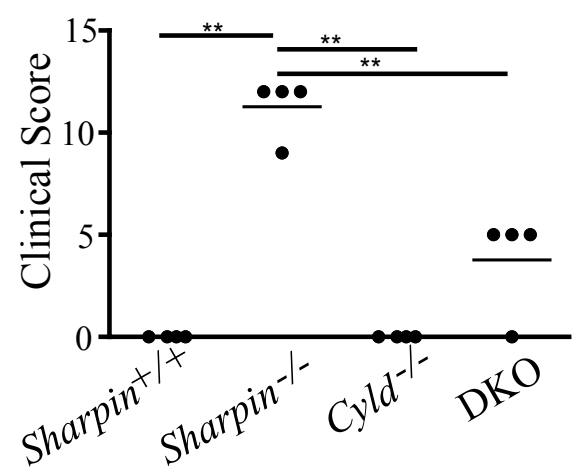

(C) Sharpin ${ }^{+/+}$

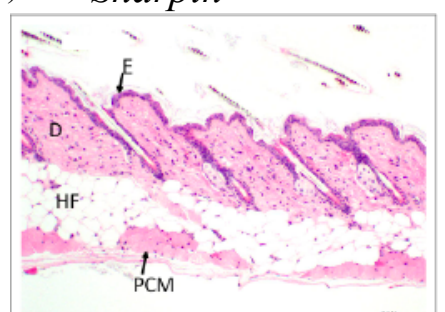

Cyld-/-

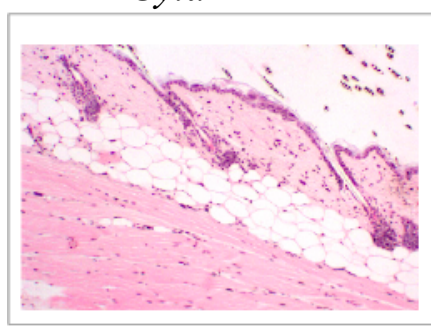

(D) Sharpin $^{+/+}$

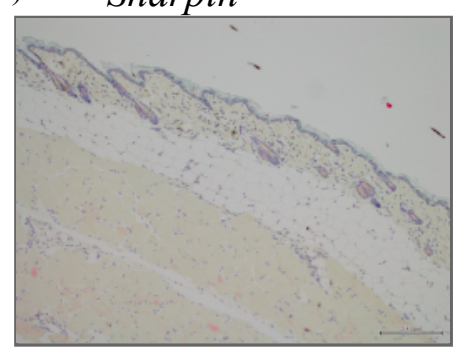

Cyld-/-

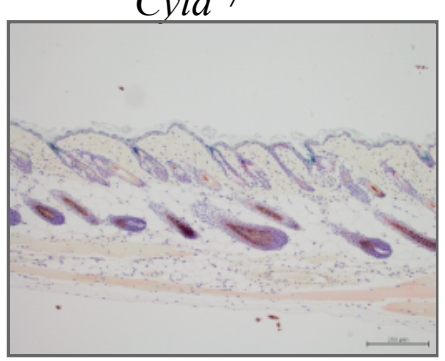

Sharpin-/-

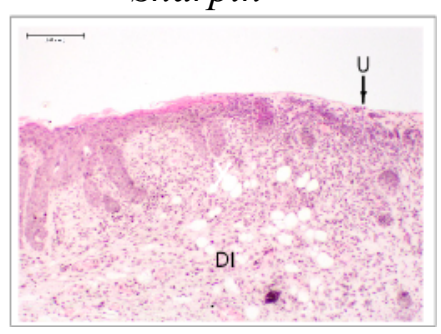

$\mathrm{DKO}$

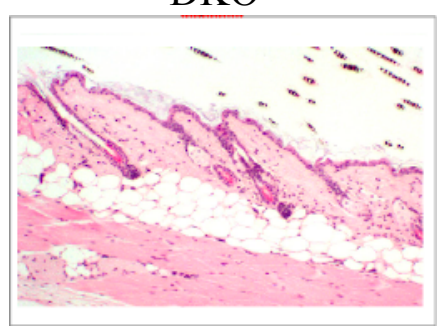

Sharpin $^{-/-}$

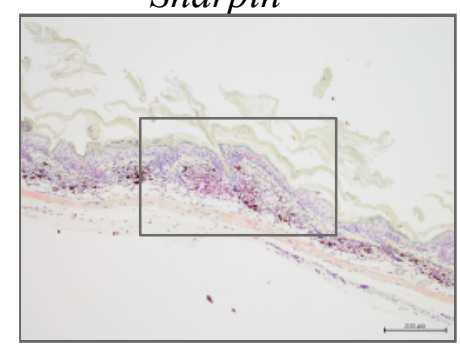

DKO

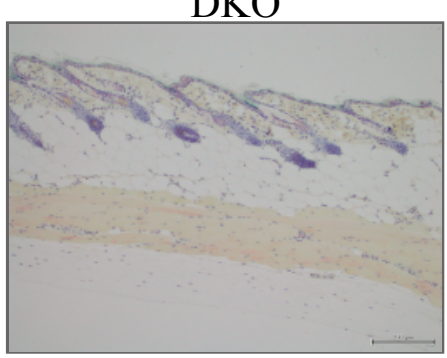

(F) Sharpin $+/+$

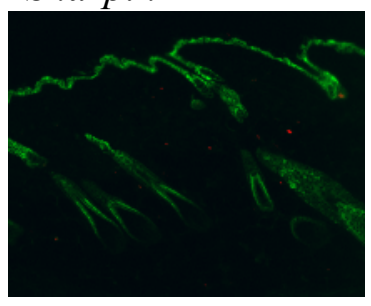

Cyld-/-

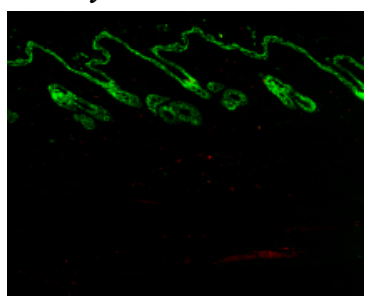

Pan-Keratin/F4/80

(E)

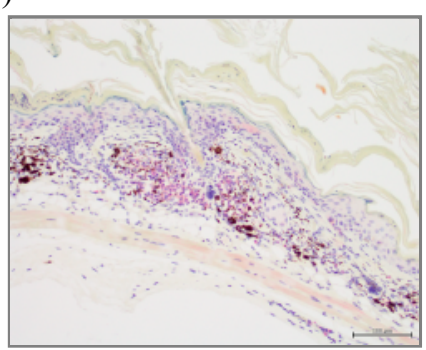

(G)

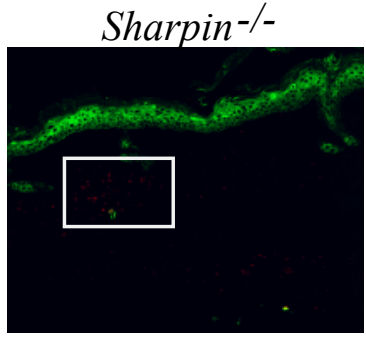

DKO
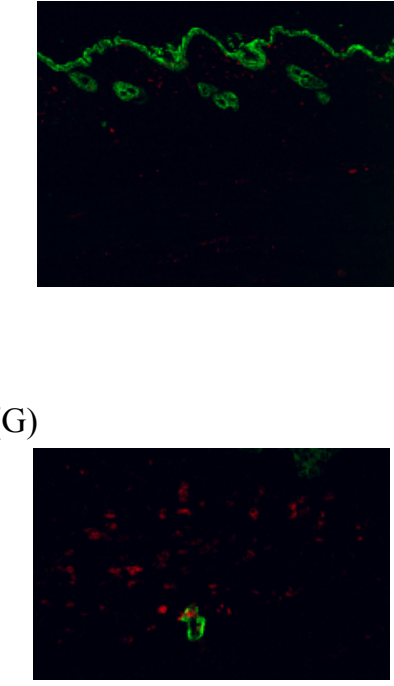

Figure 2 aioRxiv preprint doi: https://doi.org/10.1101/2020.01.27.919076; this version posted January 27,2020 . The copyright holder for this preprint
(which was not certified by peer review) is the author/funder. All rights reserved. No reuse allowed without permission.

(A)

Sharpin $^{+/+}$ Cyld ${ }^{-/}$

DKO

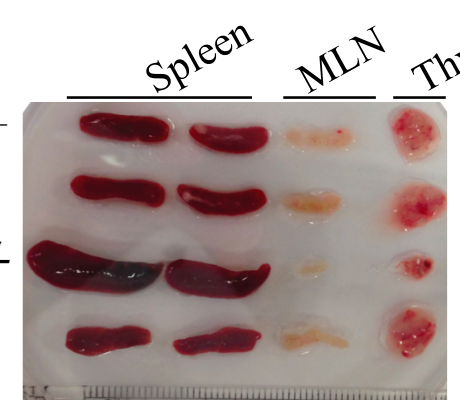
Sharpin-/-
(B)

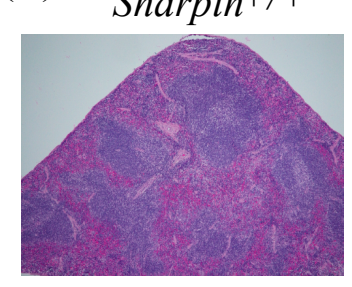

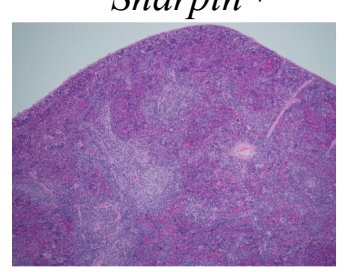

Swiss roll:

Peyer Patches:

$$
\text { Sharpin }^{+/+}
$$
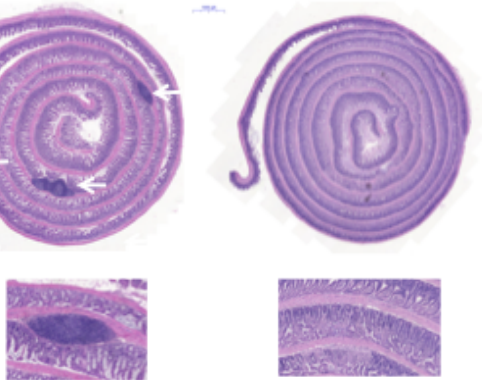

(E)
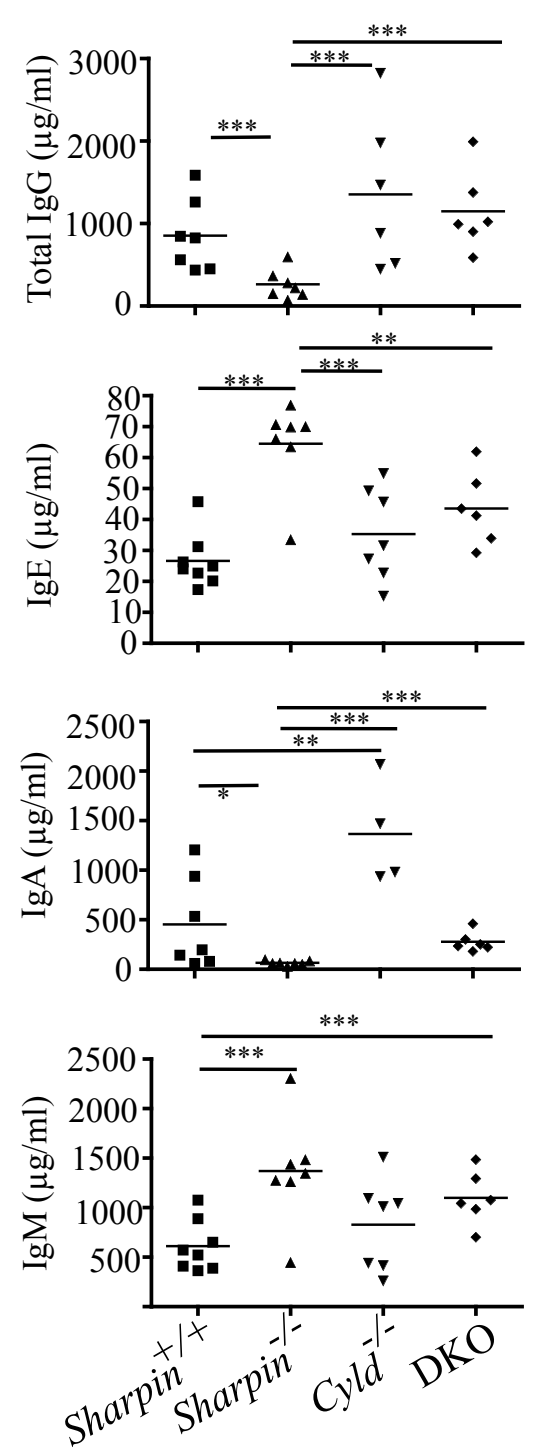

Sharpin-/-

Cyld ${ }^{-/}$

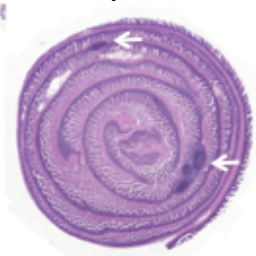

(F)

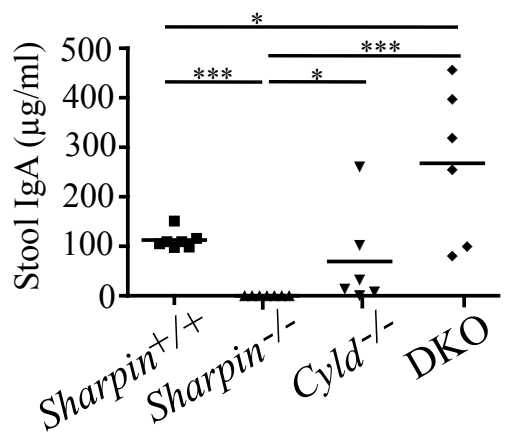

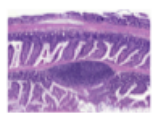

Sharpin-/-

DKO

Cyld ${ }^{-/-}$
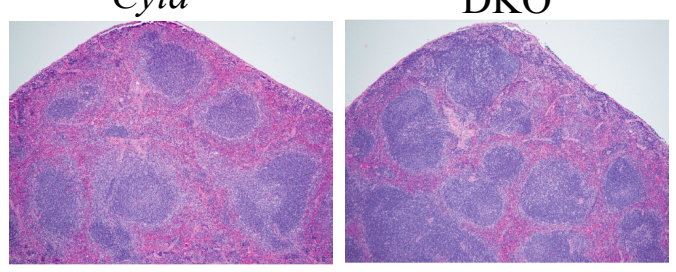

(G)
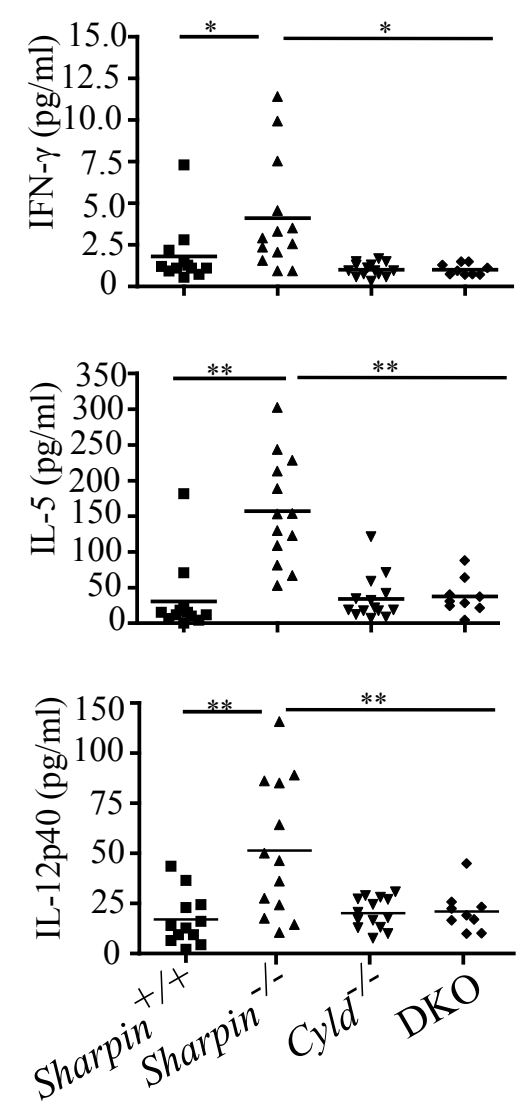
(A)

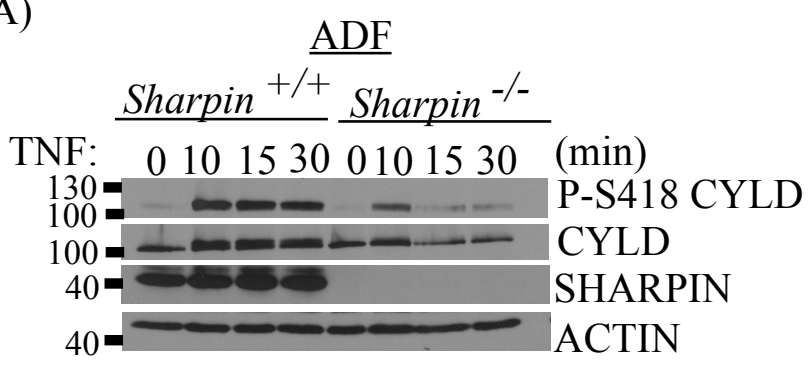

(B)

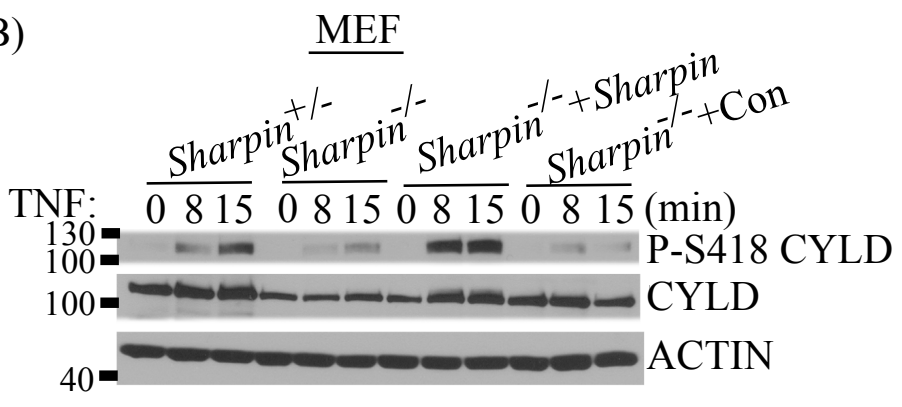

(C)

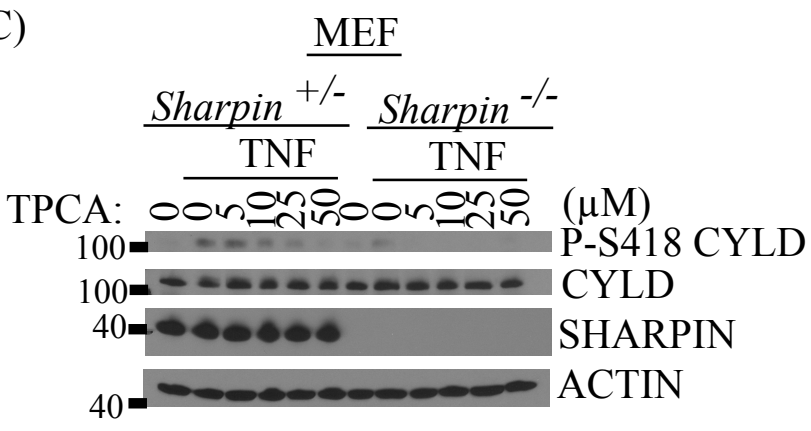

(D)

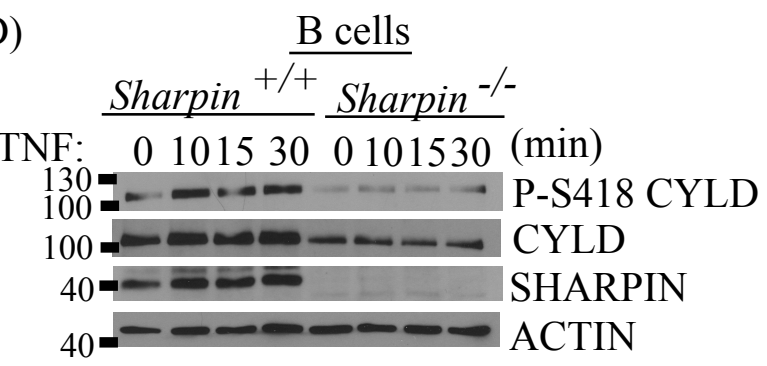

(E)

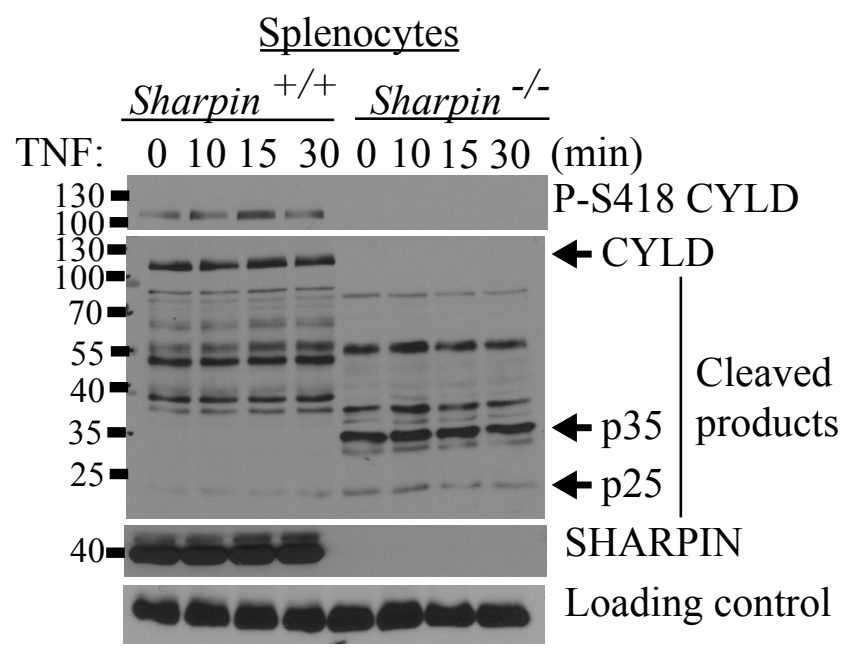


bioRxiv preprint doi: https://doi.org/10.1101/2020.01.27.919076; this version posted January 27, 2020. The copyright holder for this preprint Figure 4 (which was not certified by peer review) is the author/funder. All rights reserved. No reuse allowed without permission.

(A)

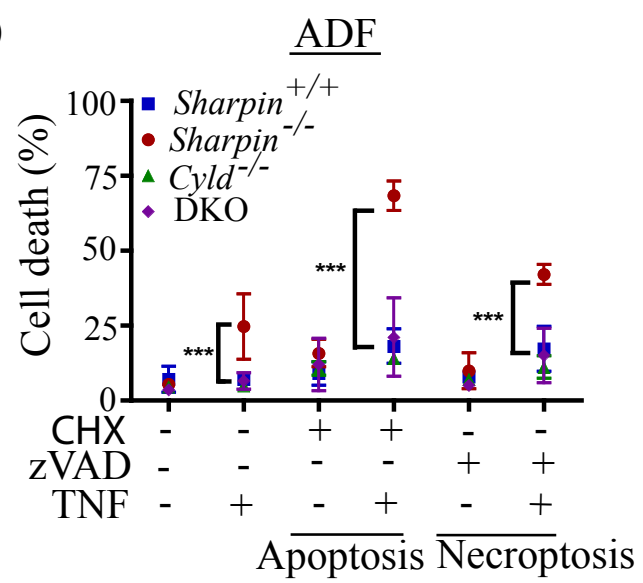

(C)

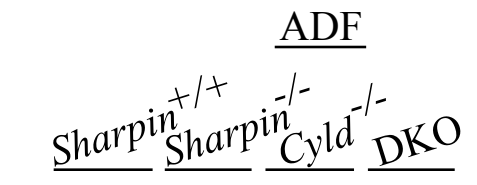

TC: $036 \overline{036} \frac{036036}{03}$ (Hour)

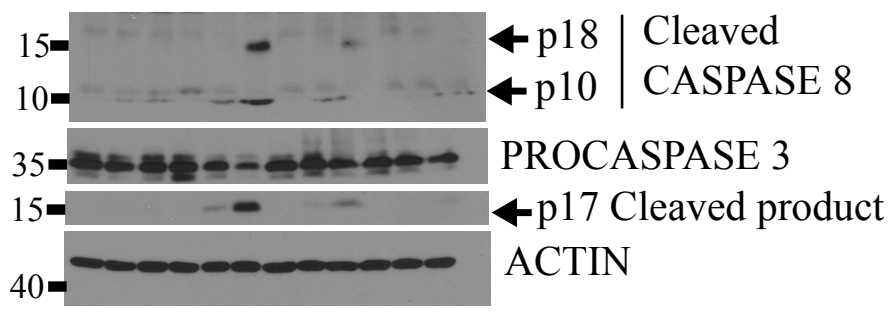

(D)

\section{ADF}

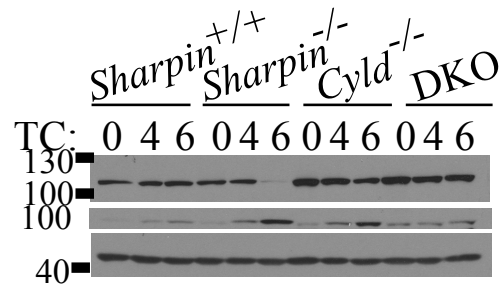

(B)

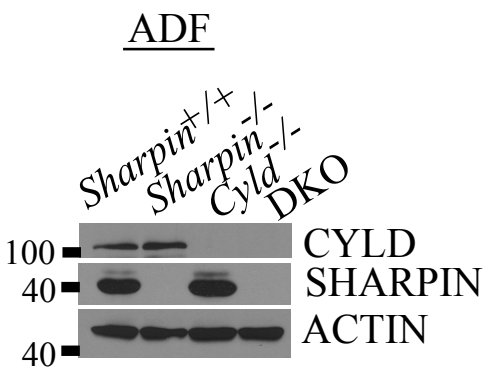

(E)

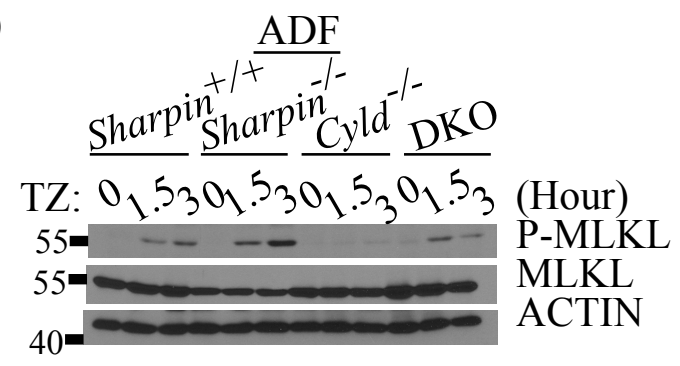

(F)

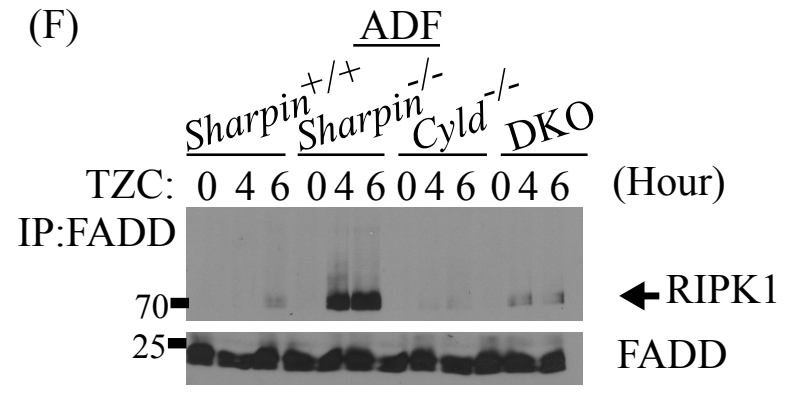

(G)

$\frac{\text { Sequences: }}{411}$

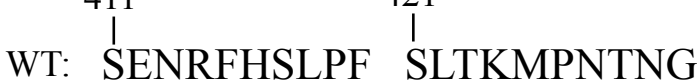
S7A: SENRFHĀLPF ĀLTKMPNTNG

PARP

4 p89 Cleaved product

ACTIN

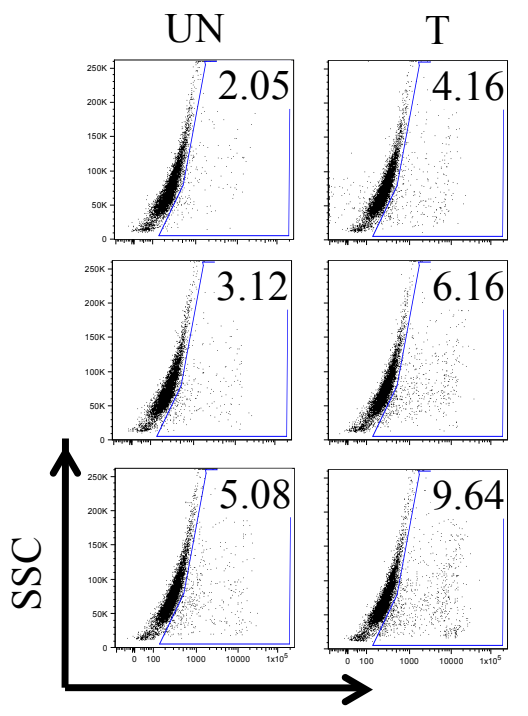

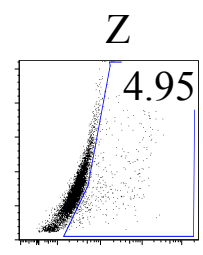
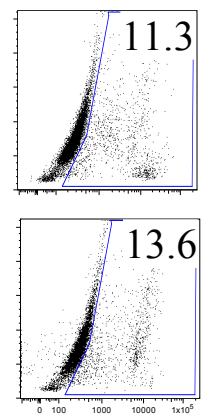

431

441

SMAHSPLSLS VQSVM

$\bar{A} M A H \bar{A} P L \bar{A} L \bar{A} \quad V Q \bar{A} V M$

PI

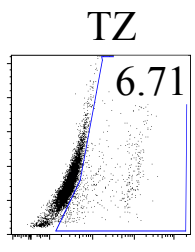

$\mathrm{CON}$

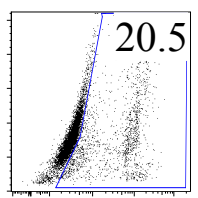

WT
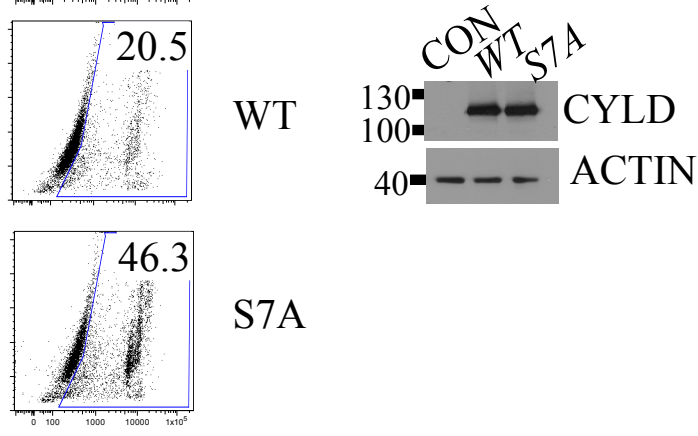
Figure 5 (which was not certified by peer review) is the author/funder. All rights reserved. No reuse allowed without permission.

(A)

BMDM

$\underline{\text { Sharpin }}+/+$ Sharpin ${ }^{-/-}$

TNF: $\begin{array}{llllllllll}0 & 10 & 15 & 30 & 0 & 10 & 1530 & \text { (min) }\end{array}$

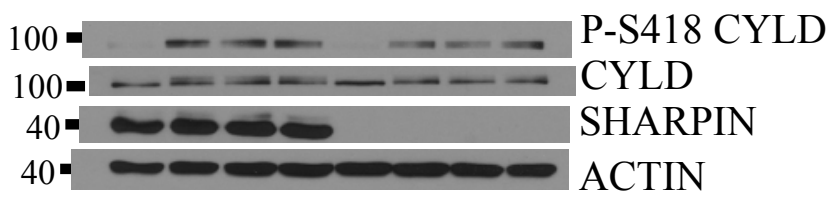

(C)

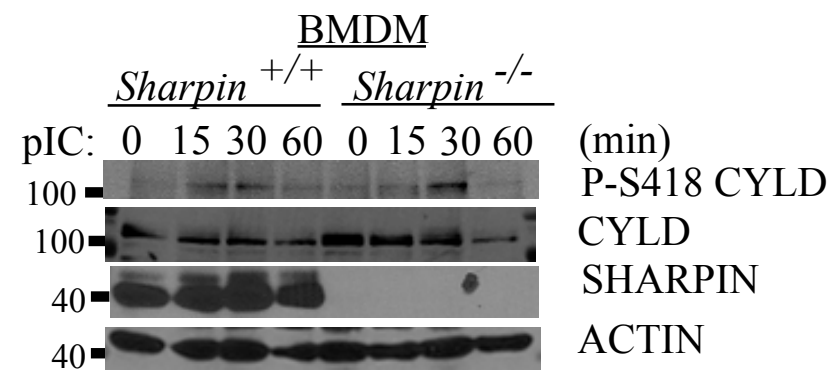

(E)

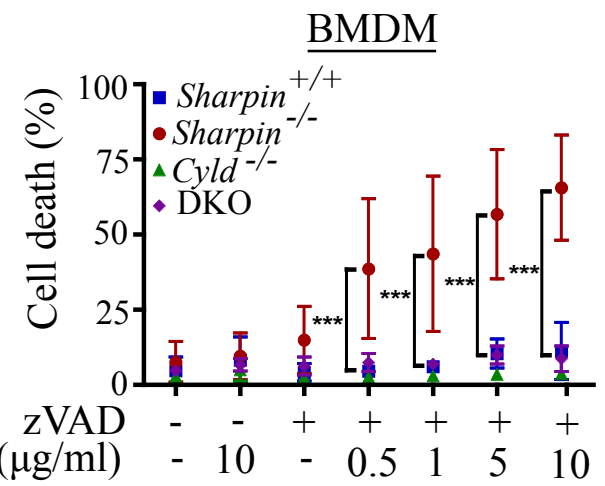

(G)
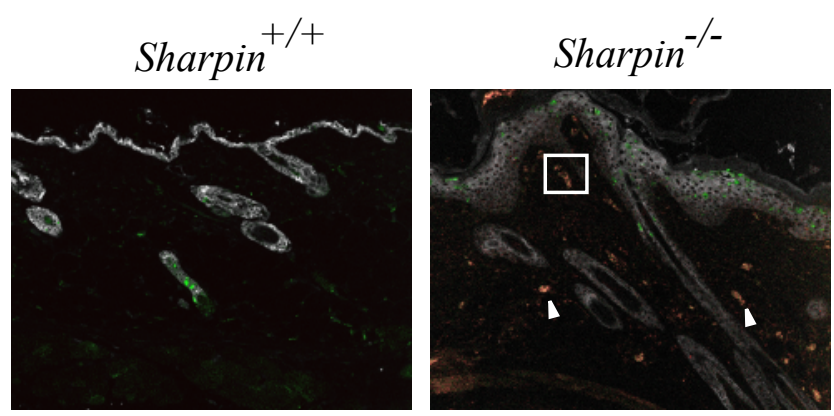

Pan-Keration F4/80 CC3

(H)

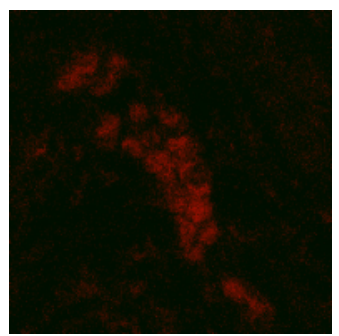

F4/80

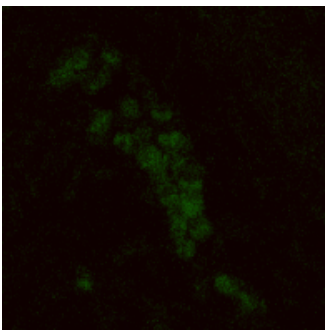

$\mathrm{CC} 3$

Overlay

(B)

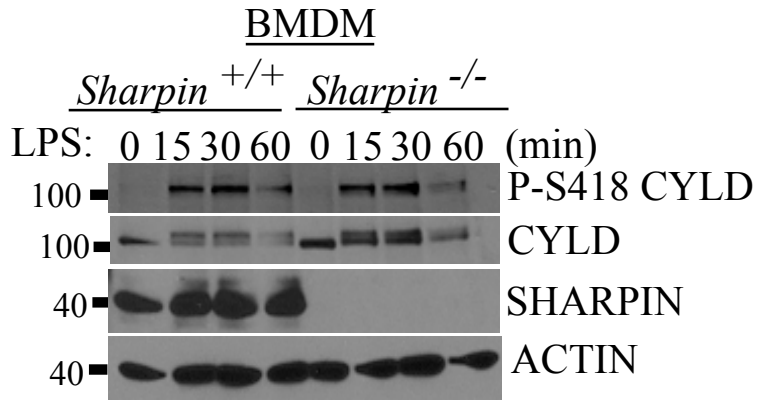

(D)

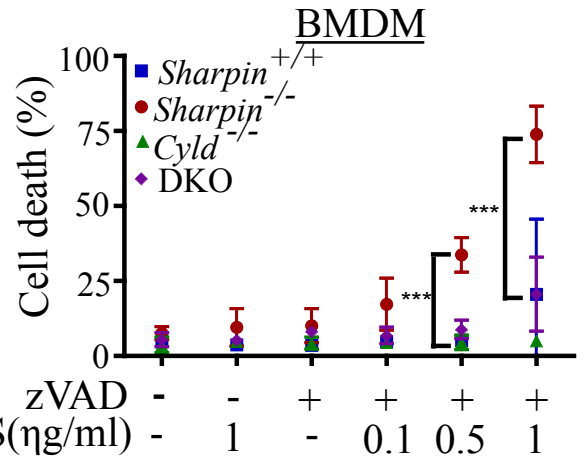

(F)

$\underline{\mathrm{BMDM}}$

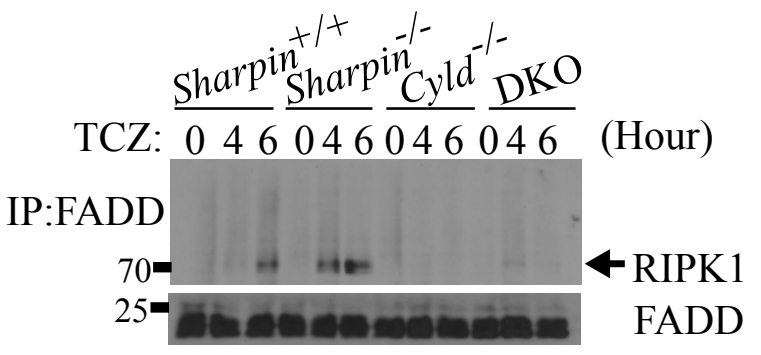

Cyld ${ }^{-/-}$

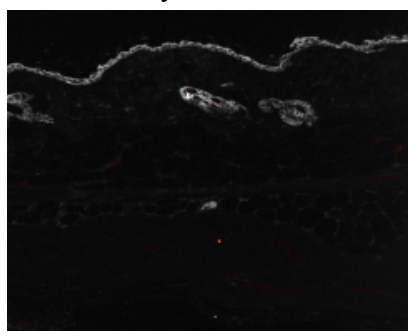

DKO

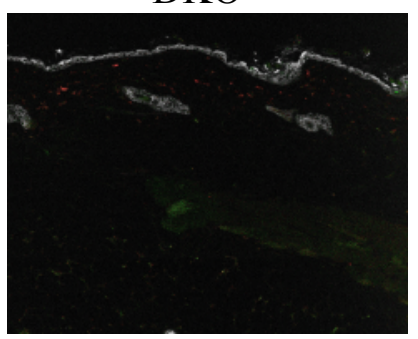

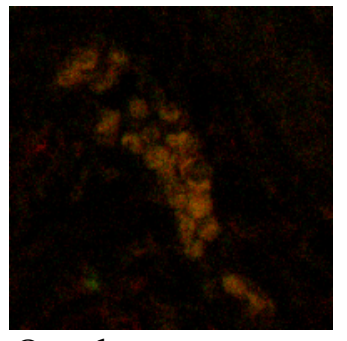


